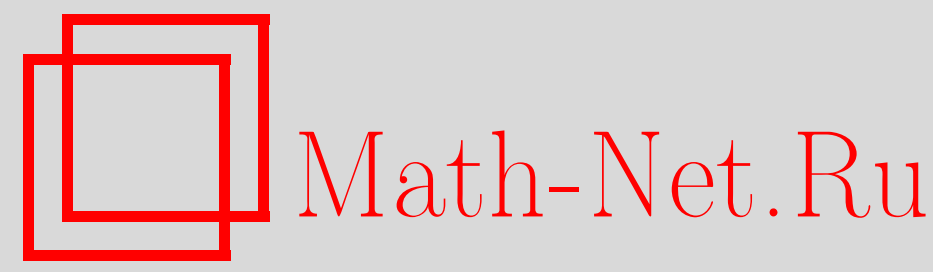

К. Пилецкас, Об асимптотике решений стационарной системы уравнений НавьеСтокса в области типа слоя, Матем. сб., 2002, том 193, номер 12, 69-104

DOI: https://doi.org/10.4213/sm700

Использование Общероссийского математического портала Math-Net.Ru подразумевает, что вы прочитали и согласны с пользовательским соглашением

http://www.mathnet.ru/rus/agreement

Параметры загрузки:

IP: 34.227 .88 .159

26 апреля 2023 г., 05:11:26 
УДК 517.9

\author{
К. Пилецкас
}

\title{
Об асимптотике решений стационарной системы уравнений Навье-Стокса в области типа слоя
}

\footnotetext{
Рассматривается стационарная система уравнений Навье-Стокса в области $\Omega \subset \mathbb{R}^{3}$, совпадаюшей для болшших $|x|$ со слоем $\Pi=\mathbb{R}^{2} \times(0,1)$. Доказывается теорема об асимптотическом поведении решений при $|x| \rightarrow \infty$. В частности, доказьвается, что для произвольных данных задачи решения, имеющие ненулевой поток через цилиндрические сечения слоя, ведут себя на бесконечности так же, как решения линейной системы Стокса.

Библиограф̆ия: 22 названия.
}

\section{§1. Введение}

Вопросы существования, единственности и асимптотического поведения решений уравнений Стокса и Навье-Стокса в областях с некомпактными границами являлись предметом исследований многих авторов. В течение последних 20 лет особое внимание уделялось задачам в областях, имеющих цилиндрические, параболические или конические выходы на бесконечность. Сушествование обобщенных решений задач Стокса и Навье-Стокса в таких областях доказано в [1]-[12]. В [13]-[16] исследовались вопросы классической разрешимости и асимптотического поведения решений этих задач. Исчерпываюшая библиография, относяшаяся $\mathrm{K}$ этой тематике, содержится в [12], [16].

Значительно меньше исследованы вопросы сушествования решений и их поведения в областях с выходами на бесконечность в виде трехмерного слоя. Существование обобщенных решений стационарной системы Навье-Стокса, имеющих заданньй поток через цилиндрические сечения слоя, доказано в [7] (см. также [11]). В недавней работе [17] исследовались задачи Стокса и Навье-Стокса в области, совпадаюшей при больших $|x|$ с бесконечньм трехмерньм слоем $\Pi=\mathbb{R}^{2} \times(0,1)$. В [17] построена последовательность точных решений однородной системы Стокса в П и доказано сушествование решений этой задачи с заданной асимптотикой на бесконечности в области $\Omega \subset \mathbb{R}^{3}$, совпадаюшей с П при больших $|x|$. Для нелинейной стационарной задачи Навье-Стокса в [17] доказано (при малых данных задачи) существование решений, асимптотически стремящихся к направленным течениям Пуазейля или Куэтта. Сушествование решений задачи Навье-Стокса с заданным потоком через цилиндрические сечения слоя получено в [17] для сколь угодно больших потоков.

В дальнейшем в работах [18], [19] было показано, что задача Стокса является фредгольмовой в шкале весовых пространств $\mathscr{V}_{\beta, \varkappa}^{l}(\Omega), l \geqslant 1,0 \leqslant \varkappa \leqslant l, \beta \in \mathbb{R}$, имеющих анизотропное распределение веса. Точное определение этих пространств 
приведено ниже в $\S 2$. Здесь лишш отметим, что при увеличении индекса $\beta$, указываюшего на наличие веса, улучшаются свойства убывания на бесконечности элементов из $\mathscr{V}_{\beta, \varkappa}^{l}(\Omega)$, индекс $l$ определяет гладкость функций, входящих в $\mathscr{V}_{\beta, \varkappa}^{l}(\Omega)$, а $\varkappa$ указывает на наличие “ступеньки” в графике функции распределения веса. В [18] также получены результаты об асимптотическом представлении на бесконечности решений задачи Стокса в слое П.

В настоящей работе исследуется асимптотическое поведение решений нелинейной стационарной системы Навье-Стокса. Работа состоит из введения и трех параграфов. В $\S 2$ вводятся основные обозначения и функциональные пространства, доказываются необходимые в дальнейшем теоремы вложения весовых пространств и формулируются используемые в работе вспомогательные результаты. В частности, в $\S 2$ приводятся результаты работ [18], [19], относящиеся к системе Стокса. В $\S 3$ доказывается теорема об асимптотическом поведении решения системы Навье-Стокса в предположении, что решение растет на бесконечности "не слишком быстро". В $\S 4$ получен основной результат работы: доказано, что решения, имеющие заданньй поток через цилиндрические сечения слоя, ведут себя на бесконечности так же, как решения линейной системы Стокса, т.е. главные члены асимптотических представлений решений линейной и нелинейной задач совпадают. Такие решения сушествуют для произвольных потоков (см. [17]), и полученный результат не требует предположений о малости данных задачи.

Отметим, что для малых данных в работе [17] доказано сушествование решения системы Навье-Стокса, имеющего на бесконечности такую же асимптотику, что и решение линейной системы Стокса. В [7] для области с двумя выходами на бесконечность, один из которых совпадает с трехмерным слоем, доказано, что при достаточно малых данных обобщенное решение системы Навье-Стокса с неограниченным интегралом Дирихле и ненулевьм потоком представимо в виде суммы решения линейной задачи Стокса и векторного поля с конечным интегралом Дирихле.

\section{§ 2. Основные обозначения и вспомогательные утверждения}

2.1. Область и функциональные пространства. Пусть $G$ - произвольная область в $\mathbb{R}^{n}$. Как обычно, через $C_{0}^{\infty}(G)$ обозначается множество всех бесконечно дифференцируемых функций с компактньми носителями в $G ; W^{l, q}(G), l \geqslant 0$ целое, $q>1,-$ пространство Соболева; $W_{\text {loc }}^{l, q}(\bar{G})$ состоит из функций, содержашихся в $W^{l, q}(K)$ для любого компакта $K \subset \bar{G} ; W^{l-1 / q, q}(G)$-пространство следов на $\partial G$ функций из $W^{l, q}(G), l \geqslant 1 ; W^{0, q}(G)=L^{q}(G) ; C^{l+\alpha}(\bar{G}), l \geqslant 0, \alpha \in(0,1),-$ пространство Гёльдера, а $C^{0}(\bar{G})$ - пространство непрерывных в $\bar{G}$ функций. Норма элемента $u$ в функциональном пространстве $V$ обозначается через $\|u ; V\|$. Пространства скалярных и векторных функций не различаются в обозначениях; из контекста будет ясно, о каком из них идет речь.

Рассмотрим в $\mathbb{R}^{3}$ область $\Omega$ с гладкой границей $\partial \Omega$, совпадаюшую вне шара $B_{R_{0}}=\left\{x \in \mathbb{R}^{3}:|x|<R_{0}\right\}$ со слоем единичной толщины

$$
\Pi=\left\{x=(y, z) \in \mathbb{R}^{3}: y=\left(y_{1}, y_{2}\right) \in \mathbb{R}^{2}, 0<z<1\right\}
$$

Обозначим через $\mathcal{V}_{\beta, \varkappa}^{l}(\Omega), l \geqslant 0,0 \leqslant \varkappa \leqslant l, \beta \in \mathbb{R}$, весовое пространство функций, 
получаемое при замькании $C_{0}^{\infty}(\bar{\Omega})$ по норме

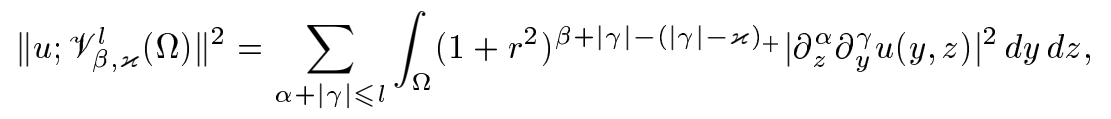

где $r=|y|=\left(y_{1}^{2}+y_{2}^{2}\right)^{1 / 2}, \alpha \geqslant 0, \gamma=\left(\gamma_{1}, \gamma_{2}\right), \gamma_{i} \geqslant 0,|\gamma|=\gamma_{1}+\gamma_{2}, \partial_{z}^{\alpha}=\partial^{\alpha} / \partial z^{\alpha}$, $\partial_{y}^{\gamma}=\partial^{|\gamma|} /\left(\partial y_{1}^{\gamma_{1}} \partial y_{2}^{\gamma_{2}}\right),(t)_{+}=(t+|t|) / 2$ - положительная часть числа $t \in \mathbb{R}$.

Пространство $\mathscr{V}_{\beta, \varkappa}^{l}(\Omega)$ является весовым пространством с анизотропным и ступенчатым распределением веса. Направление $z$ выделено в слое П как самой геометрией множества (2.1), так и строением весовой нормы (2.2): дифференцирование $\partial_{z}^{\alpha}$ не влияет на показатель степени весовой функции $\left(1+r^{2}\right)$, в то время как дифференцирование $\partial_{y}^{\gamma}$ до порядка $|\gamma| \leqslant \varkappa$ увеличивает этот показатель на $|\gamma|$. При $|\gamma|>\varkappa$ показатель степени весовой функции больше не меняется (так как $|\gamma|-(|\gamma|-\varkappa)_{+}=\varkappa$ при $\left.|\gamma|>\varkappa\right)$. Таким образом, в распределении веса возникает "ступенька". Отметим, что при $\varkappa=l$ ступенька отсутствует, а при $\varkappa=0$ для всех $|\gamma|$ имеем $|\gamma|-(|\gamma|)_{+}=0$ и, следовательно, весовой множитель при всех производных один и тот же. Наконец, заметим, что $\mathcal{V}_{0,0}^{l}(\Omega)=W^{l, 2}(\Omega)$.

Положим $L_{\beta}^{2}(\Omega)=\mathscr{V}_{\beta, 0}^{0}(\Omega)$. При $l \geqslant 1$ можно определить пространство следов $\mathcal{V}_{\beta, \varkappa}^{l-1 / 2}(\partial \Omega)$ на $\partial \Omega$ функций $v$ из $\mathscr{V}_{\beta, \varkappa}^{l}(\Omega)$. Норма в $\mathcal{V}_{\beta, \varkappa}^{l-1 / 2}(\partial \Omega)$ определяется формулой

$$
\left\|w ; \mathscr{V}_{\beta, \varkappa}^{l-1 / 2}(\partial \Omega)\right\|=\inf \left\{\left\|v ; \mathscr{V}_{\beta, \varkappa}^{l}(\Omega)\right\|: v=w \text { на } \partial \Omega\right\}
$$

Пространства $\mathcal{V}_{\beta, \varkappa}^{l}(\Omega)$ были введены в работе [18] для обоснования асимптотики решений системы Стокса в слое П. В [18] исследованы также свойства пространств $\mathscr{V}_{\beta, \varkappa}^{l}(\Omega)$ и приведена эквивалентная нормировка в пространстве следов $\mathscr{V}_{\beta, \varkappa}^{l-1 / 2}(\partial \Omega)$. Отметим, что похожие пространства вводились ранее в работе [20] при изучении задачи Неймана для эллиптического уравнения второго поря дка в П.

Обозначим через $C_{\beta}^{0}(\bar{\Omega})$ пространство непрерывных в $\bar{\Omega}$ функций, для которых конечна норма

$$
\left\|v ; C_{\beta}^{0}(\bar{\Omega})\right\|=\sup _{x \in \bar{\Omega}}\left(\left(1+r^{2}\right)^{\beta / 2}|v(x)|\right),
$$

а через $L_{\beta}^{q}(\Omega), q>1,-$ пространство функций с нормой

$$
\left\|v ; L_{\beta}^{q}(\Omega)\right\|=\left(\int_{\Omega}\left(1+r^{2}\right)^{q \beta / 2}|v(x)|^{q} d x\right)^{1 / q} .
$$

2.2. Теоремы вложения. Введем обозначения:

$$
\begin{aligned}
& \Omega(R)=\{x \in \Omega: r=|y|<R\}, \quad \Omega_{l}=\Omega\left(2^{l}\right), \quad l=0,1, \ldots ; \\
& \omega(R)=\{x \in \Omega: R<r<2 R\}, \quad \omega_{l}=\omega\left(2^{l}\right), \quad l=1,2, \ldots, \\
& \omega_{0}=\{x \in \Omega: r<2\} ; \\
& Q_{k, s}=\left\{x \in \Omega:\left|y_{1}-s\right|<1 / 2,\left|y_{2}-k\right|<1 / 2\right\}, \quad k, s \in \mathbb{Z}, \\
& Q_{k, s}^{*}=\bigcup_{i=k-1}^{k+1} \bigcup_{j=s-1}^{s+1} Q_{i, j} \supset Q_{k, s} .
\end{aligned}
$$


Лемма 2.1. Пусть $u \in W^{1,2}(\omega(R))$. Тогда $u \in L^{q}(\omega(R)), q \in[2,6], u$ справедливо неравенство

$$
\left\|u ; L^{q}(\omega(R))\right\| \leqslant c\left\|u ; W^{1,2}(\omega(R))\right\|
$$

где постоянная с не зависит от $R$.

ДокАЗАТЕЛЬСТВо. Цилиндрическое кольцо $\omega(R)$ покроем конечным числом областей $D_{j}, j=1,2, \ldots, N(R)$, таких, что

$$
c_{0} \leqslant \operatorname{mes} D_{j} \leqslant c_{1}, \quad D_{j} \subset \omega(R), \quad j=1,2, \ldots, N(R), \quad \bigcup_{j=1}^{N(R)} D_{j}=\omega(R) .
$$

В (2.7) постоянные $c_{0}$ и $c_{1}$ не зависят от $R$. Кроме того, покрытие $\left\{D_{j}\right\}_{j=1}^{N(R)}$ можно выбрать так, чтобы его кратность не зависела от $R$. Отметим, что $N(R) \rightarrow \infty$ при $R \rightarrow \infty$. В каждой $D_{j}$ имеет место оценка

$$
\left\|u ; L^{q}\left(D_{j}\right)\right\|^{q} \leqslant c\left\|u ; W^{1,2}\left(D_{j}\right)\right\|^{q}
$$

причем в силу (2.7) постоянная может быть выбрана не зависяшей от $j=$ $1, \ldots, N(R)$. Тогда

$$
\begin{aligned}
\left\|u ; L^{q}(\omega(R))\right\|^{q} & \leqslant \sum_{j=1}^{N(R)}\left\|u ; L^{q}\left(D_{j}\right)\right\|^{q} \leqslant c \sum_{j=1}^{N(R)}\left\|u ; W^{1,2}\left(D_{j}\right)\right\|^{q} \\
& =c \sum_{j=1}^{N(R)}\left(\left\|u ; W^{1,2}\left(D_{j}\right)\right\|^{2}\right)^{q / 2} \leqslant c\left(\sum_{j=1}^{N(R)}\left\|u ; W^{1,2}\left(D_{j}\right)\right\|^{2}\right)^{q / 2}
\end{aligned}
$$

(напомним, что $q / 2 \geqslant 1$ ). Поскольку кратность покрытия не зависит от $R$, то имеет место неравенство

$$
\sum_{j=1}^{N(R)}\left\|u ; W^{1,2}\left(D_{j}\right)\right\|^{2} \leqslant c\left\|u ; W^{1,2}(\omega(R))\right\|^{2} .
$$

Следовательно,

$$
\left\|u ; L^{q}(\omega(R))\right\|^{q} \leqslant c\left\|u ; W^{1,2}(\omega(R))\right\|^{q} .
$$

Лемма 2.2. Пусть $u \in W^{1,2}\left(\Omega\left(R_{1}\right) \backslash \Omega(R)\right), R_{0} \leqslant R<R_{1},\left.u\right|_{\partial \Omega}=0$. Тогда имеет место неравенство

$$
\left\|\left(1+r^{2}\right)^{\beta / 2} u ; L^{2}\left(\Omega\left(R_{1}\right) \backslash \Omega(R)\right)\right\| \leqslant\left\|\left(1+r^{2}\right)^{\beta / 2} \partial_{z} u ; L^{2}\left(\Omega\left(R_{1}\right) \backslash \Omega(R)\right)\right\| .
$$


ДокАЗАТЕЛьство. Утверждение леммы следует из одномерного неравенства Фридрихса на интервале $(0,1)$

$$
\int_{0}^{1}|u(y, z)|^{2} d z \leqslant \int_{0}^{1}\left|\partial_{z} u(y, z)\right|^{2} d z
$$

после умножения этого неравенства на $\left(1+r^{2}\right)^{\beta}$ и интегрирования по кольцу $\left\{y \in R^{2}: R<|y|<R_{1}\right\}$.

Перейдем к рассмотрению свойств весовых пространств $\mathcal{V}_{\beta, \varkappa}^{l}(\Omega)$. Непосредственно из определения нормы (2.2) вытекают следующие утверждения.

ЛЕмма 2.3. (i) Вложсения

$$
\begin{aligned}
& \mathscr{V}_{\beta, \varkappa}^{l}(\Omega) \hookrightarrow \mathcal{V}_{\beta, \varkappa}^{l-1}(\Omega), \quad l \geqslant 1, \quad 0 \leqslant \varkappa \leqslant l-1, \\
& \mathcal{V}_{\beta_{1}, \varkappa}^{l}(\Omega) \hookrightarrow \mathscr{V}_{\beta, \varkappa}^{l}(\Omega), \quad l \geqslant 0, \quad 0 \leqslant \varkappa \leqslant 1, \quad \beta_{1}>\beta,
\end{aligned}
$$

являются непрерьвными.

(ii) Пусть $v \in \mathcal{V}_{\beta, \varkappa}^{l}(\Omega), l \geqslant 1,0 \leqslant \varkappa \leqslant l, \beta \in \mathbb{R}$. Тогда $\partial_{y} v \in \mathcal{V}_{\beta+1, \varkappa-1}^{l-1}(\Omega)$, $\partial_{z} v \in \mathcal{V}_{\beta, \varkappa}^{l-1}(\Omega) u$

$$
\left\|\partial_{y} v ; \mathscr{V}_{\beta+1, \varkappa-1}^{l-1}(\Omega)\right\|+\left\|\partial_{z} v ; \mathscr{V}_{\beta, \varkappa}^{l-1}(\Omega)\right\| \leqslant c\left\|v ; \mathscr{V}_{\beta, \varkappa}^{l}(\Omega)\right\|
$$

Лемма 2.4. (i) Пусть $u \in \mathscr{V}_{\beta, \varkappa}^{l}(\Omega), l \geqslant 2,0 \leqslant \varkappa \leqslant l, \beta \in \mathbb{R}$. Eсли $\alpha+|\gamma| \leqslant l-2,|\gamma| \leqslant \varkappa-2$, то $\partial_{z}^{\alpha} \partial_{y}^{\gamma} u \in C_{\beta+1+|\gamma|}^{0}(\bar{\Omega})$ и имеет место неравенство

$$
\left\|\partial_{y}^{\gamma} \partial_{z}^{\alpha} u ; C_{\beta+1+|\gamma|}^{0}(\bar{\Omega})\right\| \leqslant c\left\|u ; \mathscr{V}_{\beta, \varkappa}^{l}(\Omega)\right\|
$$

Ecлu же $|\gamma| \geqslant \varkappa-1$, mo $\partial_{z}^{\alpha} \partial_{y}^{\gamma} u \in C_{\beta+|\gamma|-(|\gamma|-\varkappa)_{+}}^{0}(\bar{\Omega}) u$

$$
\left\|u ; C_{\beta+|\gamma|-(|\gamma|-\varkappa)_{+}}^{0}(\bar{\Omega})\right\| \leqslant c\left\|u ; \mathscr{V}_{\beta, \varkappa}^{l}(\Omega)\right\|
$$

(ii) Пусть $u \in \mathcal{V}_{\beta, \varkappa}^{l}(\Omega), l \geqslant 1, \beta \in \mathbb{R}$. Ecлu $|\gamma|+\alpha=l-1,|\gamma| \leqslant \varkappa-1$, mo $\partial_{z}^{\alpha} \partial_{y}^{\gamma} u \in L_{\beta+|\gamma|+1-2 / q}^{q}(\Omega), q \in[2,6]$, и справедлива оценка

$$
\left\|\partial_{z}^{\alpha} \partial_{y}^{\gamma} u ; L_{\beta+|\gamma|+1-2 / q}^{q}(\Omega)\right\| \leqslant c\left\|u ; \mathcal{V}_{\beta, \varkappa}^{l}(\Omega)\right\| .
$$

Ecли же $|\gamma| \geqslant \varkappa$, mo $\partial_{z}^{\alpha} \partial_{y}^{\gamma} u \in L_{\beta+|\gamma|-(|\gamma|-\varkappa)_{+}}^{q}(\Omega) u$

$$
\left\|\partial_{z}^{\alpha} \partial_{y}^{\gamma} u ; L_{\beta+|\gamma|-(|\gamma|-\varkappa)_{+}}^{q}(\Omega)\right\| \leqslant c\left\|u ; \mathcal{V}_{\beta, \varkappa}^{l}(\Omega)\right\| .
$$


ДокаЗАТЕльство. Пусть $\alpha+|\gamma| \leqslant l-2,|\gamma| \leqslant \varkappa-2$. Рассмотрим в области $\omega_{k}$ функцию $v_{\alpha \gamma}(x)=\partial_{y}^{\gamma} \partial_{z}^{\alpha} u(x)$. Введем новые переменные $(\xi, \eta)=\left(2^{-k} y, z\right)$ и положим $\widehat{v}_{\alpha \gamma}(\xi, \eta)=v_{\alpha \gamma}\left(2^{k} \xi, \eta\right)$. При такой замене область $\omega_{k}, 2^{k} \geqslant R_{0}$, переходит в стандартную область $\widehat{\omega}=\{(\xi, \eta): 1<|\xi|<2,0<\eta<1\}$. Если $\alpha+|\gamma| \leqslant l-2$, то $\widehat{v}_{\alpha \gamma} \in W^{l-\alpha-|\gamma|, 2}(\widehat{\omega}) \subset W^{2,2}(\widehat{\omega})$ и по теореме вложения Соболева

$$
\sup _{(\xi, \eta) \in \bar{\omega}}\left|\widehat{v}_{\alpha \gamma}(\xi, \eta)\right| \leqslant c\left\|\widehat{v}_{\alpha \gamma} ; W^{2,2}(\widehat{\omega})\right\| .
$$

Возврашаясь к переменным $(y, z)$, отсюда выводим

$$
\sup _{(y, z) \in \bar{\omega}_{k}}\left|\partial_{y}^{\gamma} \partial_{z}^{\alpha} u(y, z)\right| \leqslant c\left(\sum_{j+|\mu| \leqslant 2} \int_{\omega_{k}} 2^{k(2|\mu|-2)}\left|\partial_{y}^{\mu} \partial_{z}^{j} v_{\alpha \gamma}(y, z)\right|^{2} d y d z\right)^{1 / 2}
$$

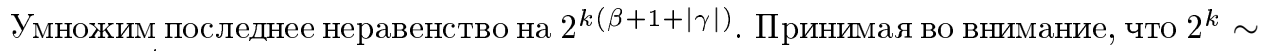
$\left(1+r^{2}\right)^{1 / 2}$ при $(y, z) \in \omega_{k}$, получаем

$$
\begin{aligned}
& \sup _{(y, z) \in \bar{\omega}_{k}}\left(\left(1+r^{2}\right)^{(\beta+1+|\gamma|) / 2}\left|\partial_{y}^{\gamma} \partial_{z}^{\alpha} u(y, z)\right|\right) \\
& \quad \leqslant c\left(\sum_{j+|\mu| \leqslant 2} \int_{\omega_{k}}\left(1+r^{2}\right)^{\beta+|\gamma|+|\mu|}\left|\partial_{y}^{\gamma+\mu} \partial_{z}^{j+\alpha} u(y, z)\right|^{2} d y d z\right)^{1 / 2} .
\end{aligned}
$$

Поскольку $|\gamma|+|\mu| \leqslant \varkappa$ величина, стоящая в правой части (2.16), не превосходит $c\left\|u ; \mathscr{V}_{\beta, \varkappa}^{l}(\Omega)\right\|$ и, следовательно,

$$
\sup _{(y, z) \in \bar{\omega}_{k}}\left(\left(1+r^{2}\right)^{(\beta+1+|\gamma|) / 2}\left|\partial_{y}^{\gamma} \partial_{z}^{\alpha} u(y, z)\right|\right) \leqslant c\left\|u ; \mathcal{Y}_{\beta, \varkappa}^{l}(\Omega)\right\| .
$$

В области $\Omega\left(R_{0}+1\right)$ по теореме вложения справедливо неравенство

$$
\sup _{x \in \overline{\Omega\left(R_{0}+1\right)}}\left|\partial_{y}^{\gamma} \partial_{z}^{\alpha} u(y, z)\right| \leqslant c\left\|\partial_{y}^{\gamma} \partial_{z}^{\alpha} u ; W^{2,2}\left(\Omega\left(R_{0}+1\right)\right)\right\| \leqslant c\left\|u ; \mathscr{V}_{\beta, \varkappa}^{l}(\Omega)\right\| .
$$

Постоянная в $(2.17)$ не зависит от $k$. Поэтому, взяв супремум по всем $k$ таким, что $2^{k} \geqslant R_{0}$, из (2.17), (2.18) получим (2.12).

Если $\alpha+|\gamma|=l-1,|\gamma| \leqslant \varkappa-1$, то из теоремы вложения следует, что $\widehat{v}_{\alpha \gamma} \in L^{q}(\widehat{\omega})$ $\forall q \in[2,6]$ и

$$
\int_{\widehat{\omega}}\left|\widehat{v}_{\alpha \gamma}\right|^{q} d \xi d \eta \leqslant c\left(\int_{\widehat{\omega}}\left(\left|\widehat{v}_{\alpha \gamma}\right|^{2}+\left|\partial_{\xi} \widehat{v}_{\alpha \gamma}\right|^{2}+\left|\partial_{\eta} \widehat{v}_{\alpha \gamma}\right|^{2}\right) d \xi d \eta\right)^{q / 2} .
$$

Переходя к координатам $(y, z)$ и умножая полученное неравенство на $2^{k q(\beta+|\gamma|+1)}$, вьводим соотношение

$$
\begin{aligned}
& \int_{\omega_{k}}\left(1+r^{2}\right)^{q(\beta+1+|\gamma|-2 / q) / 2}\left|\partial_{y}^{\gamma} \partial_{z}^{\alpha} u(y, z)\right|^{q} d y d z \\
& \quad \leqslant c\left(\sum_{|\mu|+j \leqslant 1} \int_{\omega_{k}}\left(1+r^{2}\right)^{\beta+|\gamma|+|\mu|}\left|\partial_{y}^{\gamma+\mu} \partial_{z}^{\alpha+j} u(y, z)\right|^{2} d y d z\right)^{q / 2} .
\end{aligned}
$$


Так как $c_{1} \leqslant\left(1+r^{2}\right) \leqslant c_{2}$ при $x \in \Omega\left(R_{0}+1\right)$, то в ограниченной области $\Omega\left(R_{0}+1\right)$ имеет место неравенство

$$
\begin{aligned}
& \int_{\Omega\left(R_{0}+1\right)}\left(1+r^{2}\right)^{q(\beta+1+|\gamma|-2 / q) / 2}\left|\partial_{y}^{\gamma} \partial_{z}^{\alpha} u(y, z)\right|^{q} d y d z \\
& \quad \leqslant c\left\|\partial_{y}^{\gamma} \partial_{z}^{\alpha} u ; W^{1,2}\left(\Omega\left(R_{0}+1\right)\right)\right\|^{q} \leqslant c\left\|u ; \mathscr{V}_{\beta, \varkappa}^{l}(\Omega)\right\|^{q} .
\end{aligned}
$$

Просуммируем соотношения (2.19) по $k, 2^{k} \geqslant R_{0}$. Поскольку $q / 2 \geqslant 1,|\gamma| \leqslant \varkappa-1$, в результате получим оценку

$$
\begin{aligned}
& \int_{\Omega \backslash \Omega\left(R_{0}\right)}\left(1+r^{2}\right)^{q(\beta+1+|\gamma|-2 / q) / 2}\left|\partial_{y}^{\gamma} \partial_{z}^{\alpha} u(y, z)\right|^{q} d y d z \\
& \leqslant c \sum_{k: 2^{k} \geqslant R_{0}}^{\infty}\left(\sum_{|\mu|+j \leqslant 1} \int_{\omega_{k}}\left(1+r^{2}\right)^{\beta+|\gamma|+|\mu|}\left|\partial_{y}^{\gamma+\mu} \partial_{z}^{\alpha+j} u(y, z)\right|^{2} d y d z\right)^{q / 2} \\
& \quad \leqslant c\left(\sum_{k: 2^{k} \geqslant R_{0}}^{\infty} \sum_{|\mu|+j \leqslant 1} \int_{\omega_{k}}\left(1+r^{2}\right)^{\beta+|\gamma|+|\mu|}\left|\partial_{y}^{\gamma+\mu} \partial_{z}^{\alpha+j} u(y, z)\right|^{2} d y d z\right)^{q / 2} \\
& \leqslant c\left(\sum_{|\mu|+j \leqslant 1} \int_{\Omega \backslash \Omega\left(R_{0}\right)}\left(1+r^{2}\right)^{\beta+|\gamma|+|\mu|}\left|\partial_{y}^{\gamma+\mu} \partial_{z}^{\alpha+j} u(y, z)\right|^{2} d y d z\right)^{q / 2} \\
& \leqslant c\left\|u ; \mathcal{V}_{\beta, \varkappa}^{l}(\Omega)\right\|^{q},
\end{aligned}
$$

которая совместно с предыдущим неравенством дает (2.14).

Пусть теперь $\alpha+|\gamma| \leqslant l-2,|\gamma| \geqslant \varkappa-1$. Для любой области $Q_{p, s}$ имеет место соотношение

$$
\sup _{x \in \bar{Q}_{p, s}}\left|v_{\alpha \gamma}(y, z)\right| \leqslant c\left\|v_{\alpha \gamma} ; W^{2,2}\left(Q_{p, s}\right)\right\|,
$$

причем постоянная $c$ не зависит от $p$ и $s$.

Умножим эти неравенства на $\left(1+\left(p^{2}+s^{2}\right)\right)^{\left(\beta+|\gamma|-(|\gamma|-\varkappa)_{+}\right) / 2}$. Поскольку $1+$ $\left(p^{2}+s^{2}\right) \sim\left(1+r^{2}\right)$ при $x \in Q_{p, s}$, отсюда выводим

$$
\begin{aligned}
& \sup _{x \in \bar{Q}_{p, s}}\left(\left(1+r^{2}\right)^{\left(\beta+|\gamma|-(|\gamma|-\varkappa)_{+}\right) / 2}\left|\partial_{y}^{\gamma} \partial_{z}^{\alpha} u(y, z)\right|\right)
\end{aligned}
$$

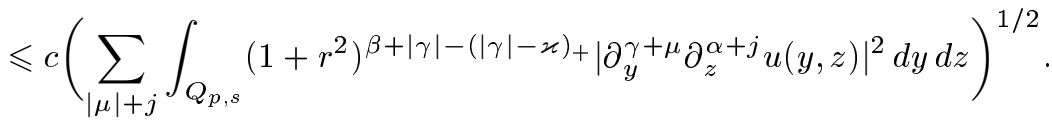

Легко видеть, что $|\gamma|-(|\gamma|-\varkappa)_{+} \leqslant|\gamma|+|\mu|-(|\gamma|+|\mu|-\varkappa)_{+} \forall|\mu| \geqslant 0$. Поэтому из (2.20) следует оценка

$$
\sup _{x \in \bar{Q}_{p, s}}\left(\left(1+r^{2}\right)^{\left(\beta+|\gamma|-(|\gamma|-\varkappa)_{+}\right) / 2}\left|\partial_{y}^{\gamma} \partial_{z}^{\alpha} u(y, z)\right|\right) \leqslant c\left\|u ; \mathcal{V}_{\beta, \varkappa}^{l}(\Omega)\right\| .
$$

Взяв в (2.21) супремум по всем $p, s \in \mathbb{Z}$, приходим к (2.13). 
Если $\alpha+|\gamma|=l,|\gamma| \geqslant \varkappa$, то по теореме вложения для любого $q \in[2,6]$ имеет место неравенство

$$
\int_{Q_{p, s}}\left|v_{\alpha \gamma}(y, z)\right|^{q} d y d z \leqslant c\left(\int_{Q_{p, s}}\left(\left|v_{\alpha \gamma}\right|^{2}+\left|\partial_{y} v_{\alpha \gamma}\right|^{2}+\left|\partial_{z} v_{\alpha \gamma}\right|^{2}\right) d y d z\right)^{q / 2},
$$

в котором постоянная $c$ не зависит от $p$ и $s$.

Умножая $(2.22)$ на $\left(1+\left(p^{2}+s^{2}\right)\right)^{q\left(\beta+|\gamma|-(|\gamma|-\varkappa)_{+}\right) / 2}$ и суммируя полученные неравенства по всем $p, s \in \mathbb{Z}$, выводим соотношение

$$
\begin{aligned}
& \int_{\Omega}\left(1+r^{2}\right)^{q\left(\beta+|\gamma|-(|\gamma|-\varkappa)_{+}\right) / 2}\left|\partial_{y}^{\gamma} \partial_{z}^{\alpha} u(y, z)\right|^{q} d y d z
\end{aligned}
$$

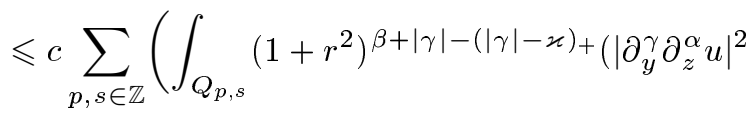

$$
\begin{aligned}
& \left.\left.+\left|\partial_{y}^{\gamma+1} \partial_{z}^{\alpha} u\right|^{2}+\left|\partial_{y}^{\gamma} \partial_{z}^{\alpha+1} u\right|^{2}\right) d y d z\right)^{q / 2} \\
& \leqslant c\left(\sum _ { p , s \in \mathbb { Z } } \int _ { Q _ { p , s } } ( 1 + r ^ { 2 } ) ^ { \beta + | \gamma | - ( | \gamma | - \varkappa ) _ { + } } \left(\left|\partial_{y}^{\gamma} \partial_{z}^{\alpha} u\right|^{2}\right.\right. \\
& \left.\left.+\left|\partial_{y}^{\gamma+1} \partial_{z}^{\alpha} u\right|^{2}+\left|\partial_{y}^{\gamma} \partial_{z}^{\alpha+1} u\right|^{2}\right) d y d z\right)^{q / 2} \\
& \leqslant c\left(\sum_{|\mu|+j \leqslant 1} \int_{\Omega}\left(1+r^{2}\right)^{\beta+|\gamma|+|\mu|-(|\gamma|+|\mu|-\varkappa)_{+}}\left|\partial_{y}^{\gamma+\mu} \partial_{z}^{\alpha+j} u\right|^{2} d y d z\right)^{q / 2} \\
& \leqslant c\left\|u ; \mathscr{V}_{\beta, \varkappa}^{l}(\Omega)\right\|^{q} \text {, }
\end{aligned}
$$

которое и доказывает неравенство (2.15).

2.3. Задача Стокса. Рассмотрим в области $\Omega$ краевую задачу для системы Стокса

$$
\begin{gathered}
-\nu \Delta \mathbf{u}+\nabla p=\mathbf{f}, \quad x \in \Omega, \\
\operatorname{div} \mathbf{u}=g, \quad x \in \Omega, \\
\mathbf{u}=\mathbf{h}, \quad x \in \partial \Omega .
\end{gathered}
$$

Здесь $\mathbf{u}=\left(\mathbf{u}^{\prime}, u_{3}\right), \mathbf{u}^{\prime}=\left(u_{1}, u_{2}\right), \nabla=\left(\partial / \partial x_{1}, \partial / \partial x_{2}, \partial / \partial x_{3}\right), \operatorname{div} \mathbf{u}=\nabla \cdot \mathbf{u}$, $\mathbf{f}=\left(\mathbf{f}^{\prime}, f_{3}\right), \mathbf{h}=\left(\mathbf{h}^{\prime}, h_{3}\right)$.

В область определения $\mathscr{D}_{\beta}^{l}(\Omega)$ оператора задачи (2.23) поместим все такие пары $(\mathbf{u}, p)$, что

$$
\left(\mathbf{u}^{\prime}, u_{3}\right) \in \mathscr{V}_{\beta+1, l}^{l+1}(\Omega) \times \mathscr{V}_{\beta+2, l-1}^{l+1}(\Omega), \quad p \in \mathscr{V}_{\beta, l}^{l}(\Omega), \quad \partial_{z} p \in \mathscr{V}_{\beta+2, l-1}^{l-1}(\Omega) .
$$

Норма в $\mathscr{D}_{\beta}^{l}(\Omega)$ определяется формулой

$$
\begin{aligned}
\left\|(\mathbf{u}, p) ; \mathscr{D}_{\beta}^{l}(\Omega)\right\|= & \left\|\mathbf{u}^{\prime} ; \mathfrak{Y}_{\beta+1, l}^{l+1}(\Omega)\right\|+\left\|u_{3} ; \mathscr{V}_{\beta+2, l-1}^{l+1}(\Omega)\right\| \\
& +\left\|p ; \mathcal{V}_{\beta, l}^{l}(\Omega)\right\|+\left\|\partial_{z} p ; \mathscr{V}_{\beta+2, l-1}^{l-1}(\Omega)\right\| .
\end{aligned}
$$


Обозначим через $\mathscr{R}_{\beta}^{l}(\Omega ; \partial \Omega)$ множество, состоящее из вектор-функций $(\mathbf{f}, g, \mathbf{h})$ таких, что $\mathbf{f}$ допускает представление в виде

$$
\mathbf{f}=\mathbf{f}_{0}+\partial_{z} \mathbf{f}_{1}+\nabla \psi
$$

и справедливы включения

$$
\mathbf{f}_{0} \in \mathscr{V}_{\beta+2, l-1}^{l-1}(\Omega), \quad \mathbf{f}_{1}^{\prime} \in \mathscr{V}_{\beta+1, l}^{l}(\Omega), \quad f_{13} \in \mathscr{V}_{\beta+2, l-1}^{l}(\Omega), \quad \psi \in \mathscr{V}_{\beta, l}^{l}(\Omega),
$$

$\partial_{z} \psi \in \mathscr{V}_{\beta+2, l-1}^{l-1}(\Omega), \quad g \in \mathscr{V}_{\beta+2, l-1}^{l}(\Omega), \quad \mathbf{h}^{\prime} \in \mathscr{V}_{\beta+1, l}^{l+1 / 2}(\partial \Omega), \quad h_{3} \in \mathscr{V}_{\beta+2, l-1}^{l+1 / 2}(\partial \Omega)$.

Норма в $\mathscr{R}_{\beta}^{l}(\Omega ; \partial \Omega)$ задается формулой

$$
\begin{aligned}
& \left\|(\mathbf{f}, g, \mathbf{h}) ; \mathscr{R}_{\beta}^{l}(\Omega ; \partial \Omega)\right\|=\inf \left\{\left\|\mathbf{f}_{0} ; \mathscr{V}_{\beta+2, l-1}^{l-1}(\Omega)\right\|+\left\|\mathbf{f}_{1}^{\prime} ; \mathscr{V}_{\beta+1, l}^{l}(\Omega)\right\|\right. \\
& \left.\quad+\left\|f_{13} ; \mathscr{V}_{\beta+2, l-1}^{l}(\Omega)\right\|+\left\|\psi ; \mathscr{V}_{\beta, l}^{l}(\Omega)\right\|+\left\|\partial_{z} \psi ; \mathscr{V}_{\beta+2, l-1}^{l-1}(\Omega)\right\|\right\} \\
& \quad+\left\|g ; \mathscr{V}_{\beta+2, l-1}^{l}(\Omega)\right\|+\left\|\mathbf{h}^{\prime} ; \mathscr{V}_{\beta+1, l}^{l+1 / 2}(\partial \Omega)\right\|+\left\|h_{3} ; \mathscr{V}_{\beta+2, l-1}^{l+1 / 2}(\partial \Omega)\right\|,
\end{aligned}
$$

где инфимум вычисляется по всем представлениям функции $\mathbf{f}$ в виде (2.25).

В работах [18], [19] были доказаны следующие утверждения.

Tеорема 2.1 (см. [18]). (i) Пусть $(\mathbf{u}, p) \in L_{\beta}^{2}(\Omega) \times L_{\beta}^{2}(\Omega), \beta \in(k, k+1)$, $k \in \mathbb{Z}$, является решением задачи Стокса (2.23) с правой частью $(\mathbf{f}, g, \mathbf{h}) \in$ $\mathscr{R}_{\beta_{1}}^{l}(\Omega ; \partial \Omega), l \geqslant 1, \beta_{1} \geqslant \beta, \beta_{1} \in(k, k+1)$. Tогдa $(\mathbf{u}, p) \in \mathscr{D}_{\beta_{1}}^{l}(\Omega) u$

$$
\left\|(\mathbf{u}, p) ; \mathscr{D}_{\beta_{1}}^{l}(\Omega)\right\| \leqslant c\left(\left\|(\mathbf{f}, g, \mathbf{h}) ; \mathscr{R}_{\beta_{1}}^{l}(\Omega ; \partial \Omega)\right\|+\left\|(\mathbf{u}, p) ; L_{\beta}^{2}(\Omega)\right\|\right) .
$$

(ii) $\Pi$ усть $(\mathbf{f}, g, \mathbf{h}) \in \mathscr{R}_{\beta+k}^{l}(\Omega ; \partial \Omega), l \geqslant 1, \beta \neq 0, \pm 1, \ldots, k \in\{1,2, \ldots\}$. Тогда решение $(\mathbf{u}, p) \in L_{\beta}^{2}(\Omega)$ задачи (2.23) допускает асимптотическое представление

$$
\left(\begin{array}{c}
\mathbf{u} \\
p
\end{array}\right)=\chi(r) \sum_{-\beta-k-1<m<-\beta-1}\left(\begin{array}{c}
c_{m}^{+} \mathbf{U}_{m}^{+}(y, z)+c_{m}^{-} \mathbf{U}_{m}^{-}(y, z) \\
c_{m}^{+} P_{m}^{+}(y)+c_{m}^{-} P_{m}^{-}(y)
\end{array}\right)+\left(\begin{array}{c}
\widetilde{\mathbf{u}} \\
\widetilde{p}
\end{array}\right)
$$

где $\chi-$ гладкая срезающая функиия, $\chi(r)=1 n p u r \geqslant 2 R_{0} u \chi(r)=0$ при $r \leqslant R_{0}$

$$
\begin{gathered}
\mathbf{U}_{m}^{ \pm}(y, z)=(2 \nu)^{-1} z(z-1) \nabla P_{m}^{ \pm}(y), \\
P_{0}^{+}(y)=1, \quad P_{0}^{-}(y)=-(2 \pi)^{-1} \ln r, \\
P_{m}^{+}(y)=(2 \pi|m|)^{-1 / 2} r^{m} \cos (m \varphi), \\
P_{m}^{-}(y)=(2 \pi|m|)^{-1 / 2} r^{m} \sin (|m| \varphi),
\end{gathered}
$$

$(r, \varphi)$ - полярные координаты в $\mathbb{R}^{2}, c_{m}^{ \pm}, m=0, \pm 1, \ldots,-$ постоянные,$(\widetilde{\mathbf{u}}, \widetilde{p}) \in$ $\mathscr{D}_{\beta+k}^{l}(\Omega)$. Кроме того, имеет место оченка

$$
\begin{array}{r}
\left\|(\widetilde{\mathbf{u}}, \widetilde{p}) ; \mathscr{D}_{\beta+k}^{l}(\Omega)\right\|+\sum_{-\beta-k-1<m<-\beta-1}\left(\left|c_{m}^{+}\right|+\left|c_{m}^{-}\right|\right) \\
\leqslant c\left(\left\|(\mathbf{f}, g, \mathbf{h}) ; \mathscr{R}_{\beta+k}^{l}(\Omega ; \partial \Omega)\right\|+\left\|(\mathbf{u}, p) ; L_{\beta}^{2}(\Omega)\right\|\right) .
\end{array}
$$

Обозначим через $\mathscr{S}_{\beta}^{l}$ оператор задачи Стокса $(2.23)$ :

$$
\mathscr{D}_{\beta}^{l}(\Omega) \ni(\mathbf{u}, p) \mapsto \mathscr{S}_{\beta}^{l}(\mathbf{u}, p)=(\mathbf{f}, g, \mathbf{h}) \in \mathscr{R}_{\beta}^{l}(\Omega ; \partial \Omega) .
$$


Tеорема 2.2 (см. [19]). (i) Пусть $l \geqslant 1$. Если $\beta$ не принадлежит множеству $\mathbb{Z}=\{0, \pm 1, \pm 2, \ldots\}$, то оператор $\mathscr{S}_{\beta}^{l}$ является фредгольмовым, т.е. множество $\mathscr{S}_{\beta}^{l} \mathscr{D}_{\beta}^{l}(\Omega)$ является замкнутым подпространством пространства $\mathscr{R}_{\beta}^{l}(\Omega ; \partial \Omega), u$

$$
\operatorname{dim} \operatorname{ker} \mathscr{S}_{\beta}^{l}<\infty, \quad \operatorname{dim} \operatorname{coker} \mathscr{S}_{\beta}^{l}<\infty
$$

Если жсе $\beta \in \mathbb{Z}$, то мнохсество значений оператора $\mathscr{S}_{\beta}^{l}$ незамкнуто в $\mathscr{R}_{\beta}^{l}(\Omega ; \partial \Omega)$.

(ii) При $\beta \geqslant-1$ оператор $\mathscr{S}_{\beta}^{l}$ является мономорфизмом, а при $\beta<-1 \mathscr{S}_{\beta}^{l}$ является эпиморфизмом. Кроме того, имеют место формуль

$$
\begin{gathered}
\operatorname{dim} \text { coker } \mathscr{S}_{\beta}^{l}=2 k+1, \quad \beta \in(k-1, k), \quad k \geqslant 0, \quad k \in \mathbb{Z} ; \\
\operatorname{dim} \operatorname{ker} \mathscr{S}_{\beta}^{l}=-2 q-1, \quad \beta \in(q-1, q), \quad q \leqslant-1, \quad q \in \mathbb{Z} ; \\
\operatorname{Ind} \mathscr{S}_{\beta}^{l}=-2 p-1, \quad \beta \in(p, p+1), \quad p \in \mathbb{Z} .
\end{gathered}
$$

Tеорема 2.3 (см. [19]). (i) Пусть $(\mathbf{u}, p) \in \mathscr{D}_{\beta}^{l}(\Omega), \beta \in(-2,-1)$, - решение задачи Стокса (2.23) с правой частью $(\mathbf{f}, g, \mathbf{h}) \in \mathscr{R}_{\beta+1}^{l}(\Omega ; \partial \Omega)$. Тогда коэффиииент $c_{0}^{-}$в асимптотической формуле (2.28) допускает интегральное представление

$$
c_{0}^{-}=-12 \nu\left(\int_{\partial \Omega} \mathbf{h} \cdot \mathbf{n} d s-\int_{\Omega} g d x\right) .
$$

(ii) Пусть $(\mathbf{u}, p) \in \mathscr{D}_{\beta}^{l}(\Omega), \beta>-1,-$ решение задачи Стокса (2.23) с правой частью $(\mathbf{f}, g, \mathbf{h}) \in \mathscr{R}_{\beta+k}^{l}(\Omega ; \partial \Omega)$. Тогда коэффициенты $c_{-m}^{ \pm}$в асимптотической формуле (2.28) допускают интегральные представления

$$
\begin{gathered}
c_{-m}^{ \pm}=-12 \nu\left(\int_{\Omega} \mathbf{f} \cdot \boldsymbol{\xi}_{m}^{ \pm} d x-\int_{\Omega} g \eta_{m}^{ \pm} d x+\int_{\partial \Omega} \mathbf{h} \cdot\left(\eta_{m}^{ \pm} \mathbf{n}-\nu \partial_{n} \boldsymbol{\xi}_{m}^{ \pm}\right) d s\right) \\
-\beta-k-1<-m<-\beta-1
\end{gathered}
$$

где $\left(\boldsymbol{\xi}_{m}^{ \pm}, \eta_{m}^{ \pm}\right)$- решения однородной задачи Стокса, допускающие асимптотические разложсения

$$
\left(\boldsymbol{\xi}_{m}^{ \pm}(x), \eta_{m}^{ \pm}(x)\right)=\left(\mathbf{U}_{m}^{ \pm}(y, z), P_{m}^{ \pm}(y)\right)+\left(\widetilde{\boldsymbol{\xi}}_{m}^{ \pm}(x), \widetilde{\eta}_{m}^{ \pm}(x)\right), \quad m=1,2, \ldots
$$

Здесь функиии $\left(\mathbf{U}_{m}^{ \pm}(y, z), P_{m}^{ \pm}(y)\right)$ заданьц формулами $(2.29),(2.30)$, а $\left(\widetilde{\boldsymbol{\xi}}_{m}^{ \pm}, \widetilde{\eta}_{m}^{ \pm}\right) \in$ $\mathscr{D}_{\gamma}^{l}(\Omega)$ с произвольным $\gamma$, удовлетворяющим условию

$$
-1<\gamma<0
$$

(Сушествование решений однородной задачи Стокса, обладающих упомянутыми выше свойствами, доказано в работе [19].) 
2.4. Оператор $\operatorname{div}$. В области $G \subset \mathbb{R}^{n}, n \geqslant 2$, рассмотрим задачу: найти вектор-функцию $\mathbf{u}(x)$, удовлетворяющую уравнению

$$
\operatorname{div} \mathbf{u}=g, \quad x \in G,
$$

и краевому условию

$$
\mathbf{u}=0, \quad x \in \partial G .
$$

Имеют место следующие утверждения.

Лемма 2.5 (см. [3]). Пусть $g \in L^{2}(G) u$

$$
\int_{G} g(x) d x=0
$$

Если $G$ - ограниченная область с липшицевой границей, то существует решение $\mathbf{u}$ задачи (2.32), (2.33) такое, что

$$
\left\|\nabla \mathbf{u} ; L^{2}(G)\right\| \leqslant c\left\|g ; L^{2}(G)\right\| .
$$

Постоянная с в (2.35) зависит от области $G$, но не зависит от $g$ и $\mathbf{u}$.

Лемма 2.6 (см. [21]). Пусть $G$ - произвольная область с границей $\partial G \in C^{l+2}$. Предположим, что функиия $g \in W^{l, 2}(G)$ и удовлетворяет условию (2.34). Eсли $\operatorname{supp} g \subset \bar{G}_{1}$, где $G_{1}$ - ограниченная подобласть области $G, G_{1} \neq G$, то существует решение $\mathbf{u} \in W^{l+1,2}(G)$ задачи (2.32), (2.33) такое, что supp $\mathbf{u} \subset \bar{G}_{2}$, где $G_{2}$ - ограниченная подобласть $G, G_{2} \neq G, G_{2} \neq G_{1} u$ $G_{1} \subset G_{2} \subset G$. Имеет место оченка

$$
\left\|\mathbf{u} ; W^{l+1,2}(G)\right\| \leqslant c\left\|g ; W^{l, 2}(G)\right\| .
$$

Постоянная с в (2.36) зависит от $G_{1}, G_{2}$, но не зависит от $G, g$ и $\mathbf{u}$.

Рассмотрим теперь задачу $(2.32),(2.33)$ в области $\omega_{k}$.

Лемма 2.7 (см. [7]). Пусть $g \in L^{2}\left(\omega_{k}\right)$,

$$
\int_{\omega_{k}} g(x) d x=0 .
$$

Тогда существует решение и задачи (2.32), (2.33), удовлетворяющее неравенству

$$
\left\|\nabla \mathbf{u} ; L^{2}\left(\omega_{k}\right)\right\| \leqslant c 2^{k}\left\|g ; L^{2}\left(\omega_{k}\right)\right\|, \quad k=1,2, \ldots .
$$

Постоянная с в (2.37) не зависит от $k$.

В завершение параграфа сформулируем результаты о решениях задачи (2.32), (2.33) в области типа слоя.

Пусть

$$
\mathscr{B}_{\beta}^{1}(\Omega)=\left\{\boldsymbol{\eta}=\left(\boldsymbol{\eta}^{\prime}, \eta_{3}\right): \boldsymbol{\eta}^{\prime} \in \mathscr{V}_{\beta, 1}^{1}(\Omega), \eta_{3} \in \mathscr{V}_{\beta+1,1}^{1}(\Omega),\left.\boldsymbol{\eta}\right|_{\partial \Omega}=0\right\} .
$$


Определим пространство $M_{\beta}^{2}(\Omega)$ как замькание множества $C_{0}^{\infty}(\Omega)$ в норме

$$
\left\|g ; M_{\beta}^{2}(\Omega)\right\|=\left(\left\|g ; L_{\beta+1}^{2}(\Omega)\right\|^{2}+\int_{1}^{\infty} r^{2 \beta-1}|G(r)|^{2} d r\right)^{1 / 2}
$$

где

$$
G(r)=\int_{1}^{r} \int_{S_{t}} g(x) d \varphi d z d t, \quad \beta<0
$$

и

$$
G(r)=-\int_{r}^{\infty} \int_{S_{t}} g(x) d \varphi d z d t, \quad \beta \geqslant 0
$$

$S_{t}=\{x \in \Omega:|y|=t\}$.

Пространство $M_{\beta}^{2}(\Omega)$ является гильбертовым со скалярньм произведением

$$
\langle g, h\rangle_{\beta}=\int_{\Omega}\left(1+r^{2}\right)^{\beta+1} g(x) h(x) d x+\int_{1}^{\infty} r^{2 \beta-1} G(r) H(r) d r .
$$

Заметим, что при $\beta>0$ нормы $\left\|g ; M_{\beta}^{2}(\Omega)\right\|$ и $\left\|g ; L_{\beta+1}^{2}(\Omega)\right\|$ эквивалентны (см. [17]).

Обозначим через $\widehat{L}_{\beta}^{2}(\Omega)$ подпространство функций из $L_{\beta}^{2}(\Omega)$, удовлетворяющих условию

$$
\int_{\Omega} g(x) d x=0
$$

и положим

$$
\begin{aligned}
& \widehat{M}_{\beta}^{2}(\Omega)=M_{\beta}^{2}(\Omega), \quad \beta<0, \\
& \widehat{M}_{\beta}^{2}(\Omega)=M_{\beta}^{2}(\Omega) \cap \widehat{L}_{\beta}^{2}(\Omega), \quad \beta=0, \\
& \widehat{M}_{\beta}^{2}(\Omega)=\widehat{L}_{\beta+1}^{2}(\Omega), \quad \beta>0 .
\end{aligned}
$$

Лемма 2.8 (см. [17]). (i) Пусть $\mathbf{u} \in \mathscr{B}_{\beta}^{1,2}(\Omega), \operatorname{mozдa~} g=\operatorname{div} \mathbf{u} \in \widehat{M}_{\beta}^{2}(\Omega) u$

$$
\left\|g ; \widehat{M}_{\beta}^{2}(\Omega)\right\| \leqslant c\left\|\mathbf{u} ; \mathscr{B}_{\beta}^{1,2}(\Omega)\right\|
$$

(ii) Для любой функции $g \in \widehat{M}_{\beta}^{2}(\Omega)$ найдется вектор-функиия $\mathbf{u} \in \mathscr{B}_{\beta}^{1,2}(\Omega)$ такая, что $\operatorname{div} \mathbf{u}=g u$

$$
\left\|\mathbf{u} ; \mathscr{B}_{\beta}^{1,2}(\Omega)\right\| \leqslant c\left\|g ; \widehat{M}_{\beta}^{2}(\Omega)\right\| .
$$

ЛЕМма 2.9 (см. [17]). Пусть $l(\eta)$ - непрерывный линейный функиионал, заданный на пространстве $\mathscr{B}_{-\mu}^{1}(\Omega), \mu<0$, и удовлетворяющий условию

$$
l(\boldsymbol{\eta})=0 \quad \forall \boldsymbol{\eta} \in \widehat{\mathscr{B}}_{-\mu}^{1}(\Omega)=\left\{\boldsymbol{\xi} \in \mathscr{B}_{-\mu}^{1}(\Omega): \operatorname{div} \boldsymbol{\xi}=0\right\}
$$

Тогда существует функиия $p \in L_{\mu-1}^{2}(\Omega)$ такая, что

$$
l(\boldsymbol{\eta})=\int_{\Omega} p \operatorname{div} \boldsymbol{\eta} d x \quad \forall \boldsymbol{\eta} \in \mathscr{B}_{-\mu}^{1}(\Omega) .
$$

При этом норма $\left\|p ; L_{\mu-1}^{2}(\Omega)\right\|$ эквивалентна норме функиионала. 


\section{§ 3. Асимптотика решений системы уравнений Навье-Стокса}

Рассмотрим в области $\Omega$ стационарную систему Навье-Стокса

$$
\begin{gathered}
-\nu \Delta \mathbf{v}+(\mathbf{v} \cdot \nabla) \mathbf{v}+\nabla p=\mathbf{f}, \quad x \in \Omega, \\
\operatorname{div} \mathbf{v}=0, \quad x \in \Omega, \\
\mathbf{v}=\mathbf{h}, \quad x \in \partial \Omega .
\end{gathered}
$$

3.1. Обобщенное решение. Под обобщенным решением задачи (3.1) будем понимать вектор-функцию $\mathbf{v} \in W_{\mathrm{loc}}^{1,2}(\bar{\Omega})$, удовлетворяющую условиям

$$
\operatorname{div} \mathbf{v}=0,\left.\quad \mathbf{v}\right|_{\partial \Omega}=\mathbf{h}
$$

и интегральному тождеству

$$
\nu \int_{\Omega} \nabla \mathbf{v} \cdot \nabla \boldsymbol{\eta} d x-\int_{\Omega}(\mathbf{v} \cdot \nabla) \boldsymbol{\eta} \cdot \mathbf{v} d x=\int_{\Omega} \mathbf{f} \cdot \boldsymbol{\eta} d x
$$

для любой вектор-функции $\boldsymbol{\eta} \in C_{0}^{\infty}(\bar{\Omega})$ такой, что $\operatorname{div} \boldsymbol{\eta}=0,\left.\boldsymbol{\eta}\right|_{\partial \Omega}=0$.

Теорема 3.1. Пусть $-2<\beta<-1, \mathbf{f} \in L_{\beta+1}^{2}(\Omega)$, а $\mathbf{v}$ является обобщенным решением задачи (3.1). Предположсим, что

$$
\mathbf{v}^{\prime} \in \mathcal{V}_{\beta+1,1}^{1}(\Omega), \quad v_{3} \in \mathcal{V}_{\beta+2,0}^{1}(\Omega) .
$$

Тогда существует функиия $p \in L_{\beta}^{2}(\Omega)$, удовлетворяющая интегральному тождеству

$$
\nu \int_{\Omega} \nabla \mathbf{v} \cdot \nabla \boldsymbol{\eta} d x-\int_{\Omega}(\mathbf{v} \cdot \nabla) \boldsymbol{\eta} \cdot \mathbf{v} d x=\int_{\Omega} p \operatorname{div} \boldsymbol{\eta} d x+\int_{\Omega} \mathbf{f} \cdot \boldsymbol{\eta} d x
$$

для любой $\boldsymbol{\eta}$ с $\left.\nabla \boldsymbol{\eta} \in L_{-\beta-1}^{2}(\Omega) u \boldsymbol{\eta}\right|_{\partial \Omega}=0$.

ДокАЗАТЕЛЬСтво. Из условия (3.4) и леммы 2.4 следует, что

$$
\nabla \mathbf{v} \in L_{\beta+1}^{2}(\Omega), \quad \mathbf{v}^{\prime} \in L_{\beta+3 / 2}^{4}(\Omega), \quad v_{3} \in L_{\beta+2}^{4}(\Omega) \subset L_{\beta+3 / 2}^{4}(\Omega)
$$

Поэтому

$$
\begin{aligned}
\left|\int_{\Omega} \nabla \mathbf{v} \cdot \nabla \boldsymbol{\eta} d x\right| & \leqslant\left\|\nabla \mathbf{v} ; L_{\beta+1}^{2}(\Omega)\right\|\left\|\nabla \boldsymbol{\eta} ; L_{-\beta-1}^{2}(\Omega)\right\|, \\
\left|\int_{\Omega} \mathbf{f} \cdot \boldsymbol{\eta} d x\right| & \leqslant\left\|\mathbf{f} ; L_{\beta+1}^{2}(\Omega)\right\|\left\|\boldsymbol{\eta} ; L_{-\beta-1}^{2}(\Omega)\right\|, \\
\left|\int_{\Omega}(\mathbf{v} \cdot \nabla) \boldsymbol{\eta} \cdot \mathbf{v} d x\right| & \leqslant\left\|\mathbf{v} ; L_{\beta+3 / 2}^{4}(\Omega)\right\|^{2}\left\|\nabla \boldsymbol{\eta} ; L_{-2 \beta-3}^{2}(\Omega)\right\| \\
& \leqslant c\left\|\mathbf{v} ; L_{\beta+3 / 2}^{4}(\Omega)\right\|^{2}\left\|\nabla \boldsymbol{\eta} ; L_{-\beta-1}^{2}(\Omega)\right\| .
\end{aligned}
$$

При выводе последнего неравенства воспользовались тем, что $-2 \beta-3<-\beta-1$ при $\beta \in(-2,-1)$. 
Таким образом, интегральное тождество (3.3) по непрерывности можно распространить на функции $\boldsymbol{\eta}$, удовлетворяющие условиям

$$
\nabla \boldsymbol{\eta} \in L_{-\beta-1}^{2}(\Omega), \quad \operatorname{div} \boldsymbol{\eta}=0,\left.\quad \boldsymbol{\eta}\right|_{\partial \Omega}=0
$$

Очевидно, что множество функций, удовлетворяющих условиям (3.6), содержит пространство $\widehat{\mathscr{B}}_{-\beta-1}^{1}(\Omega)$. Рассмотрим функционал

$$
l(\boldsymbol{\eta})=\nu \int_{\Omega} \nabla \mathbf{v} \cdot \nabla \boldsymbol{\eta} d x-\int_{\Omega}(\mathbf{v} \cdot \nabla) \boldsymbol{\eta} \cdot \mathbf{v} d x-\int_{\Omega} \mathbf{f} \cdot \boldsymbol{\eta} d x
$$

заданньй на $\boldsymbol{\eta} \in \widehat{\mathscr{B}}_{-\beta-1}^{1}(\Omega)$. Поскольку v удовлетворяет интегральному тождеству $(3.3) \forall \boldsymbol{\eta} \in \widehat{\mathscr{B}}_{-\beta-1}^{1}(\Omega)$, то

$$
l(\boldsymbol{\eta})=0 \quad \forall \boldsymbol{\eta} \in \widehat{\mathscr{B}}_{-\beta-1}^{1}(\Omega) .
$$

В силу леммы 2.9 сушествует функция $p \in L_{\beta}^{2}(\Omega)$ такая, что

$$
l(\boldsymbol{\eta})=\int_{\Omega} p \operatorname{div} \boldsymbol{\eta} d x \quad \forall \boldsymbol{\eta} \in \widehat{\mathscr{B}}_{-\beta-1}^{1}(\Omega) .
$$

Теорема доказана.

3.2. Оценка нелинейного члена. Для доказательства теоремы об асимптотическом поведении решений задачи (3.1) нам потребуются вспомогательные неравенства, позволяющие оценить слагаемое $(\mathbf{v} \cdot \nabla) \mathbf{v}$.

Лемма 3.1. Пусть $u \in \mathcal{V}_{\beta_{1}+1, l}^{l+1}(\Omega), v \in \mathcal{V}_{\beta_{2}+2, l-1}^{l}(\Omega), l \geqslant 1$.

Тогда при $l=1: w=u \cdot v \in L_{\beta_{1}+\beta_{2}+11 / 3}^{2}(\Omega) u$

$$
\left\|w ; L_{\beta_{1}+\beta_{2}+11 / 3}^{2}(\Omega)\right\| \leqslant c\left\|u ; \mathscr{V}_{\beta_{1}+1,1}^{2}(\Omega)\right\|\left\|v ; \mathscr{V}_{\beta_{2}+2,0}^{1}(\Omega)\right\|
$$

$n p u l \geqslant 2: w \in \mathscr{V}_{\beta_{1}+\beta_{2}+4, l-1}^{l-1}(\Omega) u$

$$
\left\|w ; \mathcal{V}_{\beta_{1}+\beta_{2}+4, l-1}^{l-1}(\Omega)\right\| \leqslant c\left\|u ; \mathcal{V}_{\beta_{l}+1, l}^{l+1}(\Omega)\right\|\left\|v ; \mathcal{V}_{\beta_{2}+2, l-1}^{l}(\Omega)\right\|
$$

ДокаЗАТЕльство. Пусть $u \in \mathscr{V}_{\beta_{1}+1,1}^{2}(\Omega), v \in \mathcal{V}_{\beta_{2}+2,0}^{1}(\Omega)$. В силу леммы 2.4 (ii) (см. (2.14), (2.15)) имеет место оценка

$$
\begin{aligned}
& \int_{\Omega}\left(1+r^{2}\right)^{\beta_{1}+\beta_{2}+11 / 3}|u|^{2}|v|^{2} d x \\
& \quad \leqslant c\left(\int_{\Omega}\left(1+r^{2}\right)^{3\left(\beta_{1}+5 / 3\right)}|u|^{6} d x\right)^{1 / 3}\left(\int_{\Omega}\left(1+r^{2}\right)^{3\left(\beta_{2}+2\right) / 2}|v|^{3} d x\right)^{2 / 3} \\
& \quad \leqslant c\left\|u ; \mathscr{V}_{\beta_{1}+1,1}^{2}(\Omega)\right\|^{2}\left\|v ; \mathcal{V}_{\beta_{2}+2,0}^{1}(\Omega)\right\|^{2}
\end{aligned}
$$

Рассмотрим случай $l \geqslant 2$. Надо оценить норму

$$
\left\|w ; \mathcal{V}_{\beta_{1}+\beta_{2}+4, l-1}^{l-1}(\Omega)\right\|=\left(\sum_{r=0}^{l-1} \sum_{k=0}^{r} \int_{\Omega}\left(1+r^{2}\right)^{\beta_{1}+\beta_{2}+4+(r-k)}\left|\partial_{y}^{r-k} \partial_{z}^{k} w\right|^{2} d x\right)^{1 / 2}
$$


где $\partial_{y}^{s}=\sum_{|\gamma|=s} \partial_{y}^{\gamma}$. Имеем

$$
\left|\partial_{y}^{r-k} \partial_{z}^{k}(u \cdot v)\right| \leqslant c\left|\sum_{p=0}^{k} \sum_{j=0}^{r-k} \partial_{y}^{j} \partial_{z}^{p} u \partial_{y}^{r-k-j} \partial_{z}^{k-p} v\right|, \quad r=0,1, \ldots, l-1 .
$$

Пусть $j \leqslant l-2$. Тогда в силу $(2.12)$

$$
\begin{aligned}
& \int_{\Omega}\left(1+r^{2}\right)^{\beta_{1}+\beta_{2}+4+(r-k)}\left|\partial_{y}^{j} \partial_{z}^{p} u\right|^{2}\left|\partial_{y}^{r-k-j} \partial_{z}^{k-p} v\right|^{2} d x \\
& \quad \leqslant c\left\|\partial_{y}^{j} \partial_{z}^{p} u ; C_{\beta_{1}+2+j}^{0}(\bar{\Omega})\right\|^{2} \int_{\Omega}\left(1+r^{2}\right)^{\beta_{2}+2+(r-j-k)}\left|\partial_{y}^{r-j-k} \partial_{z}^{k-p} v\right|^{2} d x \\
& \quad \leqslant c\left\|u ; \mathcal{V}_{\beta_{1}+1, l}^{l+1}(\Omega)\right\|^{2}\left\|v ; \mathfrak{V}_{\beta_{2}+2, l-1}^{l}(\Omega)\right\|^{2} .
\end{aligned}
$$

Пусть $r=l-1, k=0, j=l-1$. При $l \geqslant 2$ из включения $v \in \mathscr{V}_{\beta_{2}+2, l-1}^{l}(\Omega) \subset$ $\mathscr{V}_{\beta_{2}+2,1}^{2}(\Omega)$ следует, что $v \in L_{\beta_{2}+5 / 2}^{4}(\Omega)$ (см. (2.14)). Поэтому

$$
\begin{aligned}
& \int_{\Omega}\left(1+r^{2}\right)^{\beta_{1}+\beta_{2}+4+(l-1)}\left|\partial_{y}^{l-1} u\right|^{2}|v|^{2} d x \\
& \quad \leqslant\left(\int_{\Omega}\left(1+r^{2}\right)^{2\left(\beta_{1}+3 / 2+l-1\right)}\left|\partial_{y}^{l-1} u\right|^{4} d x\right)^{1 / 2}\left(\int_{\Omega}\left(1+r^{2}\right)^{2 \beta_{2}+5}|v|^{4} d x\right)^{1 / 2} \\
& \quad \leqslant c\left\|u ; \mathcal{V}_{\beta_{1}+1, l}^{l+1}(\Omega)\right\|^{2}\left\|v ; \mathcal{V}_{\beta_{2}+2, l-1}^{l}(\Omega)\right\|^{2} .
\end{aligned}
$$

Таким образом,

$$
\begin{aligned}
& \sum_{r=0}^{l-1} \sum_{k=0}^{r} \int_{\Omega}\left(1+r^{2}\right)^{\beta_{1}+\beta_{2}+4+(r-k)}\left|\partial_{y}^{r-k} \partial_{z}^{k} w\right|^{2} d x \\
& \quad \leqslant c \sum_{r=0}^{l-1} \sum_{k=0}^{r} \sum_{p=0}^{k} \sum_{j=0}^{r-k} \int_{\Omega}\left(1+r^{2}\right)^{\beta_{1}+\beta_{2}+4+(r-k)}\left|\partial_{y}^{j} \partial_{z}^{p} u\right|^{2}\left|\partial_{y}^{r-k-j} \partial_{z}^{k-p} v\right|^{2} d x \\
& \quad \leqslant c\left\|u ; \mathscr{V}_{\beta_{1}+1, l}^{l+1}(\Omega)\right\|^{2}\left\|v ; \mathscr{V}_{\beta_{2}+2, l-1}^{l}(\Omega)\right\|^{2} .
\end{aligned}
$$

Лемма доказана.

Лемма 3.2. Пусть $u \in \mathscr{V}_{\beta_{1}+1, l}^{l+1}(\Omega), v \in \mathscr{V}_{\beta_{2}+2, l-1}^{l}(\Omega), l \geqslant 1 . \quad$ Тогда $w=$ $u \cdot v \in \mathscr{V}_{\beta_{1}+\beta_{2}+3, l}^{l}(\Omega) u$

$$
\left\|w ; \mathscr{V}_{\beta_{1}+\beta_{2}+3, l}^{l}(\Omega)\right\| \leqslant c\left\|u ; \mathscr{V}_{\beta_{1}+1, l}^{l+1}(\Omega)\right\|\left\|v ; \mathscr{V}_{\beta_{2}+2, l-1}^{l}(\Omega)\right\| .
$$

ДокАЗАТЕЛЬСтво. Рассмотрим норму

$$
\begin{aligned}
\left\|w ; \mathscr{V}_{\beta_{1}+\beta_{2}+3, l}^{l}(\Omega)\right\|^{2}= & \sum_{r=0}^{l-1} \sum_{k=0}^{r} \int_{\Omega}\left(1+r^{2}\right)^{\beta_{1}+\beta_{2}+3+(r-k)}\left|\partial_{y}^{r-k} \partial_{z}^{k} w\right|^{2} d x \\
& +\sum_{k=0}^{l} \int_{\Omega}\left(1+r^{2}\right)^{\beta_{1}+\beta_{2}+3+(l-k)}\left|\partial_{y}^{l-k} \partial_{z}^{k} w\right|^{2} d x .
\end{aligned}
$$


В силу леммы 3.1 первое слагаемое в правой части (3.10) оценивается через

$$
c\left\|u ; \mathscr{V}_{\beta_{1}+1, l}^{l+1}(\Omega)\right\|^{2}\left\|v ; \mathscr{V}_{\beta_{2}+2, l-1}^{l}(\Omega)\right\|^{2} .
$$

Рассмотрим второе слагаемое. Поскольку

$$
\left|\partial_{y}^{l-k} \partial_{z}^{k} w\right| \leqslant c\left|\sum_{p=0}^{k} \sum_{j=0}^{l-k} \partial_{y}^{j} \partial_{z}^{p} u \partial_{y}^{l-k-j} \partial_{z}^{k-p} v\right|
$$

то согласно (2.12) при $j+p \leqslant l-1$ имеем

$$
\begin{aligned}
& \int_{\Omega}\left(1+r^{2}\right)^{\beta_{1}+\beta_{2}+3+(l-k)}\left|\partial_{y}^{j} \partial_{z}^{p} u\right|^{2}\left|\partial_{y}^{l-k-j} \partial_{z}^{k-p} v\right|^{2} d x \\
& \leqslant c\left\|\partial_{y}^{j} \partial_{z}^{p} u ; C_{\beta_{1}+2+j}^{0}(\bar{\Omega})\right\|^{2} \int_{\Omega}\left(1+r^{2}\right)^{\beta_{2}+1+(l-j-k)}\left|\partial_{y}^{l-j-k} \partial_{z}^{k-p} v\right|^{2} d x \\
& \leqslant c\left\|u ; \mathcal{V}_{\beta_{1}+1, l}^{l+1}(\Omega)\right\|^{2} \int_{\Omega}\left(1+r^{2}\right)^{\beta_{2}+2+l-j-k-(l-j-k-(l-1))_{+}}\left|\partial_{y}^{l-j-k} \partial_{z}^{k-p} v\right|^{2} d x \\
& \leqslant c\left\|u ; \mathscr{V}_{\beta_{1}+1, l}^{l+1}(\Omega)\right\|^{2}\left\|v ; \mathscr{V}_{\beta_{2}+2, l-1}^{l}(\Omega)\right\|^{2}
\end{aligned}
$$

(здесь воспользовались тем, что $\beta_{2}+1+l-j-k \leqslant \beta_{2}+2+l-j-k-(l-j-$ $\left.k-(l-1))_{+}\right)$.

$$
\begin{aligned}
& \text { При } j+p=l \text { имеем } k=p, l-k-j=0 \text { и } \\
& \int_{\Omega}\left(1+r^{2}\right)^{\beta_{1}+\beta_{2}+3+(l-p)}\left|\partial_{y}^{l-p} \partial_{z}^{p} u\right|^{2}|v|^{2} d x \\
& \quad \leqslant\left(\int_{\Omega}\left(1+r^{2}\right)^{2\left(\beta_{1}+1+(l-p)\right)}\left|\partial_{y}^{l-p} \partial_{z}^{p} u\right|^{4} d x\right)^{1 / 2}\left(\int_{\Omega}\left(1+r^{2}\right)^{2\left(\beta_{2}+2\right)}|v|^{4} d x\right)^{1 / 2} \\
& \quad \leqslant c\left\|u ; \mathscr{V}_{\beta_{1}+1, l}^{l+1}(\Omega)\right\|^{2}\left\|v ; \mathscr{V}_{\beta_{2}+2, l-1}^{l}(\Omega)\right\|^{2} .
\end{aligned}
$$

При выводе последнего неравенства воспользовались (2.15) для оценки интеграла, содержащего $|v|^{4}$; для оценки интеграла от $\left|\partial_{y}^{l-p} \partial z^{p} u\right|^{4}$ воспользовались (2.15) при $p=0$ и (2.14) при $p \geqslant 1$.

Аналогично леммам 3.1, 3.2 доказывается

Лемма 3.3. Пусть $u \in \mathcal{V}_{\beta_{1}+2, l-1}^{l+1}(\Omega), v \in \mathcal{V}_{\beta_{2}+1, l}^{l}(\Omega)$. Тогда

(i) $n p u l=1: w=u \cdot v \in L_{\beta_{1}+\beta_{2}+11 / 3}^{2}(\Omega) u$

$$
\left\|w ; L_{\beta_{1}+\beta_{2}+11 / 3}^{2}(\Omega)\right\| \leqslant c\left\|u ; \mathscr{V}_{\beta_{1}+2,0}^{2}(\Omega)\right\|\left\|v ; \mathscr{V}_{\beta_{1}+1,1}^{1}(\Omega)\right\|
$$

$n p u l \geqslant 2: w \in \mathscr{V}_{\beta_{1}+\beta_{2}+4, l-1}^{l-1}(\Omega) u$

$$
\left\|w ; \mathcal{V}_{\beta_{1}+\beta_{2}+4, l-1}^{l-1}(\Omega)\right\| \leqslant c\left\|u ; \mathcal{V}_{\beta_{1}+2, l-1}^{l+1}(\Omega)\right\|\left\|v ; \mathcal{V}_{\beta_{2}+1, l}^{l}(\Omega)\right\|
$$

(ii) для всех $l \geqslant 1: w \in \mathscr{V}_{\beta_{1}+\beta_{2}+3, l}^{l}(\Omega) u$

$$
\left\|w ; \mathscr{V}_{\beta_{1}+\beta_{2}+3, l}^{l}(\Omega)\right\| \leqslant c\left\|u ; \mathscr{V}_{\beta_{1}+2, l-1}^{l+1}(\Omega)\right\|\left\|v ; \mathscr{V}_{\beta_{2}+1, l}^{l}(\Omega)\right\|
$$


ЛЕмма 3.4. Пусть $\mathbf{u}=\left(\mathbf{u}^{\prime}, u_{3}\right) \in \mathscr{V}_{\beta_{1}+1, l}^{l+1}(\Omega) \times \mathscr{V}_{\beta_{1}+2, l-1}^{l+1}(\Omega), \mathbf{v}=\left(\mathbf{v}^{\prime}, v_{3}\right) \in$ $\mathscr{V}_{\beta_{2}+1, l}^{l+1}(\Omega) \times \mathcal{V}_{\beta_{2}+2, l-1}^{l+1}(\Omega)$.

Тогда при $l \geqslant 2:(\mathbf{u} \cdot \nabla) \mathbf{v} \in \mathscr{V}_{\beta_{1}+\beta_{2}+4, l-1}^{l-1}(\Omega) u$

$$
\begin{aligned}
& \left\|(\mathbf{u} \cdot \nabla) \mathbf{v} ; \mathscr{V}_{\beta_{1}+\beta_{2}+4, l-1}^{l-1}(\Omega)\right\| \leqslant c\left\|\mathbf{u} ; \mathscr{V}_{\beta_{1}+1, l}^{l+1}(\Omega) \times \mathscr{V}_{\beta_{1}+2, l-1}^{l+1}(\Omega)\right\| \\
& \quad \times\left\|\mathbf{v} ; \mathscr{V}_{\beta_{2}+1, l}^{l+1}(\Omega) \times \mathscr{V}_{\beta_{2}+2, l-1}^{l+1}(\Omega)\right\| \equiv c A(\mathbf{u}, \mathbf{v})
\end{aligned}
$$

nрu $l=1:(\mathbf{u} \cdot \nabla) \mathbf{v} \in L_{\beta_{1}+\beta_{2}+11 / 3}^{2}(\Omega) u$

$$
\left\|(\mathbf{u} \cdot \nabla) \mathbf{v} ; L_{\beta_{1}+\beta_{2}+11 / 3}^{2}(\Omega)\right\| \leqslant c A(\mathbf{u} ; \mathbf{v}) ;
$$

наконеи, при всех $l \geqslant 1:(\mathbf{u} \cdot \nabla) \mathbf{v} \in \mathscr{V}_{\beta_{1}+\beta_{2}+3, l}^{l}(\Omega) u$

$$
\left\|(\mathbf{u} \cdot \nabla) \mathbf{v} ; \mathscr{V}_{\beta_{1}+\beta_{2}+3, l}^{l}(\Omega)\right\| \leqslant c A(\mathbf{u} ; \mathbf{v}) .
$$

ДокАЗАТЕЛЬСТво. Вектор-функцию $\mathbf{M}=(\mathbf{u} \cdot \nabla) \mathbf{v}$ можно представить в виде

$$
\mathbf{M}=\left(\begin{array}{c}
\mathbf{M}_{1}^{\prime}+\mathbf{M}_{2}^{\prime} \\
M_{31}+M_{32}
\end{array}\right)=\left(\begin{array}{c}
\left(\mathbf{u}^{\prime} \cdot \nabla^{\prime}\right) \mathbf{v}^{\prime}+u_{3} \partial_{z} \mathbf{v}^{\prime} \\
\left(\mathbf{u}^{\prime} \cdot \nabla^{\prime}\right) v_{3}+u_{3} \partial_{z} v_{3}
\end{array}\right)
$$

Из включения $\mathbf{v}^{\prime} \in \mathscr{V}_{\beta_{2}+1, l}^{l+1}(\Omega)$ следует, что $\nabla^{\prime} \mathbf{v}^{\prime} \in \mathscr{V}_{\beta_{2}+2, l-1}^{l}(\Omega)$. Поэтому в силу лемм $3.1,3.2$

$$
\begin{aligned}
\mathbf{M}_{1}^{\prime}= & \left(\mathbf{u}^{\prime} \cdot \nabla^{\prime}\right) \mathbf{v}^{\prime} \in \mathcal{V}_{\beta_{1}+\beta_{2}+4, l-1}^{l-1}(\Omega), \quad l \geqslant 2 ; \\
& \mathbf{M}_{1}^{\prime} \in L_{\beta_{1}+\beta_{2}+11 / 3}^{2}(\Omega), \quad l=1 ; \\
& \mathbf{M}_{1}^{\prime} \in \mathscr{V}_{\beta_{1}+\beta_{2}+3, l}^{l}(\Omega), \quad l \geqslant 1 .
\end{aligned}
$$

Далее, $u_{3} \in \mathscr{V}_{\beta_{1}+2, l-1}^{l+1}(\Omega), \partial_{z} \mathbf{v}^{\prime} \in \mathscr{V}_{\beta_{2}+1, l}^{l}(\Omega)$ и согласно лемме 3.3

$$
\begin{gathered}
\mathbf{M}_{2}^{\prime}=u_{3} \partial_{z} \mathbf{v}^{\prime} \in \mathscr{V}_{\beta_{1}+\beta_{2}+4, l-1}^{l-1}(\Omega), \quad l \geqslant 2 \\
\mathbf{M}_{2}^{\prime} \in L_{\beta_{1}+\beta_{2}+11 / 3}^{2}(\Omega), \quad l=1 \\
\mathbf{M}_{2}^{\prime} \in \mathscr{V}_{\beta_{1}+\beta_{2}+3, l}^{l}(\Omega), \quad l \geqslant 1 .
\end{gathered}
$$

Кроме того, так как

$$
\begin{gathered}
\nabla^{\prime} v_{3} \in \mathscr{V}_{\beta_{2}+3, l-2}^{l}(\Omega) \subset \mathscr{V}_{\beta_{2}+2, l-1}^{l}(\Omega), \\
\partial_{z} v_{3} \in \mathscr{V}_{\beta_{2}+2, l-1}^{l}(\Omega) \\
u_{3} \in \mathscr{V}_{\beta_{1}+2, l-1}^{l+1}(\Omega) \subset \mathscr{V}_{\beta_{1}+1, l}^{l+1}(\Omega)
\end{gathered}
$$

из лемм $3.1-3.3$ заключаем, что

$$
\begin{aligned}
M_{3}=M_{13}+M_{23}= & \left(\mathbf{u}^{\prime} \cdot \nabla^{\prime}\right) v_{3}+u_{3} \partial_{z} v_{3} \in \mathscr{V}_{\beta_{1}+\beta_{2}+4, l-1}^{l-1}(\Omega), \quad l \geqslant 2 ; \\
& M_{3} \in L_{\beta_{1}+\beta_{2}+11 / 3}^{2}(\Omega), \quad l=1 ; \\
& M_{3} \in \mathscr{V}_{\beta_{1}+\beta_{2}+3, l}^{l}(\Omega), \quad l \geqslant 1 .
\end{aligned}
$$

Все упомянутые включения сопровождаются соответствующими неравенствами, из которых и вытекают утверждения леммы. 
3.3. Асимптотика решений. Докажем теперь теорему об асимптотике и дифференциальных свойствах решений задачи (3.1), растущих на бесконечности "не слишком быстро".

ТЕОРЕМА 3.2. Пусть $\mathbf{v}$ - обобщенное решение задачи (3.1) с правой частью

$$
(\mathbf{f}, 0, \mathbf{h}) \in \mathscr{R}_{\beta_{1}}^{l}(\Omega ; \partial \Omega), \quad l \geqslant 2, \quad \beta_{1} \in(-1,0) .
$$

Предположим, что

$$
\mathbf{v}^{\prime} \in \mathscr{V}_{\beta+1,2}^{3}(\Omega), \quad v_{3} \in \mathscr{V}_{\beta+2,1}^{3}(\Omega), \quad \beta \in(-2,-1)
$$

Тогда существует функиия $p \in L_{\beta}^{2}(\Omega)$ такая, что пара $(\mathbf{v}, p)$ удовлетворяет интегральному тождеству (3.5). Кроме того, имеют место асимптотические представления

$$
\begin{aligned}
& \mathbf{v}(y, z)=\chi(r) c_{0}^{-} \mathbf{U}_{0}^{-}(y, z)+\widetilde{\mathbf{v}}(y, z) \\
& p(y, z)=c_{0}^{+}+\chi(r) c_{0}^{-} P_{0}^{-}(y)+\widetilde{p}(y, z)
\end{aligned}
$$

где $\chi-$ гладкая срезающая функиия, $\chi(r)=0$ при $r \leqslant R_{0}, \chi(r)=1$ nри $r \geqslant 2 R_{0}$, $P_{0}^{-}(y)=-(2 \pi)^{-1} \ln r, \mathbf{U}_{0}^{-}(y, z)=(2 \nu)^{-1} z(z-1) \nabla P_{0}^{-}(y), c_{0}^{ \pm}-$постоянные, причем

$$
c_{0}^{-}=12 \nu \int_{\partial \Omega} \mathbf{h} \cdot \mathbf{n} d s=-12 \nu \int_{S_{t}} \mathbf{v}^{\prime} \cdot \mathbf{n}^{\prime} d s
$$

определяет поток вектора скорости $\mathbf{v}$ через иилиндрические сечения $S_{t}=$ $\{x \in \Omega:|y|=t\}$ слоя П (n - единичный вектор внешней нормали $\kappa \partial \Omega, \mathbf{n}^{\prime}-$ единичный вектор нормали $\left.\kappa S_{t}\right),(\widetilde{\mathbf{v}}, \widetilde{p}) \in \mathscr{D}_{\beta_{1}}^{l}(\Omega)$.

ДокАЗАтЕльство. Сушествование функции $p \in L_{\beta}^{2}(\Omega)$, удовлетворяющей тождеству (3.5), следует из теоремы 3.1. Кроме того, обобшенное решение $(\mathbf{v}, p) \in$ $W_{\mathrm{loc}}^{l+1,2}(\bar{\Omega}) \times W_{\mathrm{loc}}^{l, 2}(\bar{\Omega})$ и удовлетворяет системе $(3.1)$ почти всюду в $\Omega$.

Положим $\beta=-2+\delta_{0}, \delta_{0} \in(0,1)$. Возможны три случая:
a) $\beta+\delta_{0}>\beta_{1}$,
b) $-1<\beta+\delta_{0}<\beta_{1}$,
c) $-2<\beta+\delta_{0} \leqslant-1$.

В силу леммы 3.4 из (3.18) следует, что $(\mathbf{v} \cdot \nabla) \mathbf{v} \in \mathscr{V}_{2 \delta_{0}, 1}^{1}(\Omega)=\mathscr{V}_{\left(\beta+\delta_{0}\right)+2,1}^{1}(\Omega)$. В случае а) рассмотрим $(\mathbf{v}, p) \in L_{\beta+1}^{2}(\Omega) \times L_{\beta}^{2}(\Omega) \subset L_{\beta}^{2}(\Omega) \times L_{\beta}^{2}(\Omega)$ как решение линейной задачи Стокса $(2.23)$ с правой частью $(\mathbf{f}-(\mathbf{v} \cdot \nabla) \mathbf{v}, 0, \mathbf{h}) \in \mathscr{R}_{\beta_{1}}^{2}(\Omega ; \partial \Omega)$. Применяя теорему 2.1 (ii), заключаем, что $(\mathbf{v}, p)$ допускает асимптотические представления $(3.19),(3.20)$ с $(\widetilde{\mathbf{v}}, \widetilde{p}) \in \mathscr{D}_{\beta_{1}}^{2}(\Omega)$.

В случае b) $(\mathbf{f}-(\mathbf{v} \cdot \nabla) \mathbf{v}, 0, \mathbf{h}) \in \mathscr{R}_{\beta+\delta_{0}}^{2}(\Omega ; \partial \Omega), \beta+\delta_{0} \in\left(-1, \beta_{1}\right)$. Поэтому согласно теореме 2.1 (ii) $(\mathbf{v}, p)$ удовлетворяет $(3.19),(3.20)$ c $(\widetilde{\mathbf{v}}, \widetilde{p}) \in \mathscr{D}_{\beta+\delta_{0}}^{2}(\Omega)$. Следовательно,

$$
\widetilde{\mathbf{v}}^{\prime} \in \mathscr{V}_{\beta+\delta_{0}+1,2}^{3}(\Omega), \quad \widetilde{v}_{3} \in \mathscr{V}_{\beta+\delta_{0}+2,1}^{3}(\Omega)
$$

и из леммы 3.4 следует, что $(\widetilde{\mathbf{v}} \cdot \nabla) \widetilde{\mathbf{v}} \in \mathcal{V}_{\left(\beta+3 \delta_{0}\right)+2,1}^{1}(\Omega)$. Подставляя представления $(3.19),(3.20)$ в систему (3.1), получаем задачу

$$
\begin{gathered}
-\nu \Delta \widetilde{\mathbf{v}}+\nabla \widetilde{p}=\mathbf{f}+\mathbf{f}_{0}+\mathbf{f}_{1}(\widetilde{\mathbf{v}})-(\widetilde{\mathbf{v}} \cdot \nabla) \widetilde{\mathbf{v}} \equiv \mathbf{F}, \quad x \in \Omega, \\
\operatorname{div} \widetilde{\mathbf{v}}=g_{0}, \quad x \in \Omega, \\
\widetilde{\mathbf{v}}=\mathbf{h}, \quad x \in \partial \Omega,
\end{gathered}
$$


где

$$
\begin{gathered}
\mathbf{f}_{0}=c_{0}^{-}\left[\nu \Delta\left(\chi \mathbf{U}_{0}^{-}\right)-\nabla\left(\chi P_{0}^{-}\right)\right]-\left(c_{0}^{-}\right)^{2}\left(\chi \mathbf{U}_{0}^{-} \cdot \nabla\right)\left(\chi \mathbf{U}_{0}^{-}\right), \\
g_{0}=-c_{0} \operatorname{div}\left(\chi \mathbf{U}_{0}^{-}\right), \\
\mathbf{f}_{1}(\widetilde{\mathbf{v}})=c_{0}^{-}\left[\left(\chi \mathbf{U}_{0}^{-} \cdot \nabla\right) \widetilde{\mathbf{v}}+(\widetilde{\mathbf{v}} \cdot \nabla)\left(\chi \mathbf{U}_{0}^{-}\right)\right] .
\end{gathered}
$$

Поскольку пара $\left(\mathbf{U}_{0}^{-}, P_{0}^{-}\right)$удовлетворяет однородной системе Стокса $(2.23)$ в слое П, то

$$
\begin{gathered}
\operatorname{supp}\left[\nu \Delta\left(\chi \mathbf{U}_{0}^{-}\right)-\nabla\left(\chi P_{0}^{-}\right)\right] \subset \operatorname{supp} \chi \subset\{x \in \Omega: 1<r<2\}, \\
\operatorname{supp} g_{0} \subset\{x \in \Omega: 1<r<2\} .
\end{gathered}
$$

Кроме того, используя соотношения

$$
\left|\partial_{y}^{\gamma} \partial_{z}^{\alpha} \mathbf{U}_{0}^{-\prime}(y, z)\right|^{2} \sim \frac{1}{\left(1+r^{2}\right)^{1+|\gamma|}} \quad \text { при } r \rightarrow \infty, \quad U_{03}^{-}(y, z) \equiv 0
$$

и включения (3.21), находим, что $\left(\chi \mathbf{U}_{0}^{-} \cdot \nabla\right)\left(\chi \mathbf{U}_{0}^{-}\right) \in \mathscr{V}_{\beta_{1}+2, l}^{l}(\Omega)$ для любого $\beta_{1}<0, \mathbf{f}_{1}(\widetilde{\mathbf{v}}) \in \mathcal{V}_{\left(\beta+\delta_{0}+1\right)+2,1}^{2}(\Omega) \subset \mathscr{V}_{\beta_{1}+2,1}^{1}(\Omega)$ (напомним, что $\beta+\delta_{0} \in\left(-1, \beta_{1}\right)$, $\left.\beta_{1} \in(-1,0)\right)$.

Таким образом,

$$
\begin{aligned}
& \left(\mathbf{F}, g_{0}, \mathbf{h}\right) \in \mathscr{R}_{\beta_{1}}^{2}(\Omega ; \partial \Omega), \quad \text { если } \beta+3 \delta_{0} \geqslant \beta_{1}, \\
& \left(\mathbf{F}, g_{0}, \mathbf{h}\right) \in \mathscr{R}_{\beta+3 \delta_{0}}^{2}(\Omega ; \partial \Omega), \text { если } \beta+3 \delta_{0}<\beta_{1} .
\end{aligned}
$$

По теореме 2.1 (i) решение $(\widetilde{\mathbf{v}}, \widetilde{p})$ задачи (3.22) в первом случае попадает в пространство $\mathscr{D}_{\beta_{1}}^{2}(\Omega)$, а во втором - в пространство $\mathscr{D}_{\beta+3 \delta_{0}}^{2}(\Omega)$. Если $\beta+3 \delta_{0}<\beta_{1}$, то продолжаем итерации. В силу леммы 3.4 имеем $(\widetilde{\mathbf{v}} \cdot \nabla) \widetilde{\mathbf{v}} \in \mathscr{V}_{\left(\beta+7 \delta_{0}\right)+2,1}^{1}(\Omega)$. Если $\beta+7 \delta_{0} \geqslant \beta_{1}$, то отсюда заключаем, что $(\widetilde{\mathbf{v}}, \widetilde{p}) \in \mathscr{D}_{\beta_{1}}^{2}(\Omega)$. Если же $\beta+7 \delta_{0}<\beta_{1}$, то, продолжая этот процесс, за конечное число шагов получим

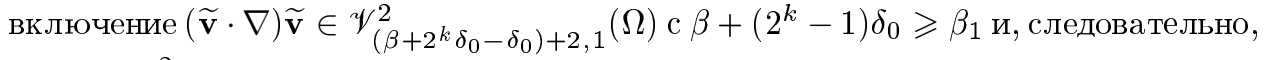
$(\widetilde{\mathbf{v}}, \widetilde{p}) \in \mathscr{D}_{\beta_{1}}^{2}(\Omega)$.

Повысим теперь гладкость решения $(\widetilde{\mathbf{v}}, \tilde{p})$. Так как $\beta_{1} \in(-1,0)$, то, применяя лемму 3.4 (см. (3.16)), получаем включение $(\widetilde{\mathbf{v}} \cdot \nabla) \widetilde{\mathbf{v}} \in \mathscr{V}_{2 \beta_{1}+3,2}^{2}(\Omega) \subset \mathscr{V}_{\beta_{1}+2,2}^{2}(\Omega)$. Тогда $\left(\mathbf{F}, g_{0}, \mathbf{h}\right) \in \mathscr{R}_{\beta_{1}}^{3}(\Omega ; \partial \Omega)$ и из теоремы 2.1 (i) выводим $(\widetilde{\mathbf{v}}, \widetilde{p}) \in \mathscr{D}_{\beta_{1}}^{3}(\Omega)$. Продолжая эти рассуждения, за конечное число шагов получим $(\widetilde{\mathbf{v}}, \widetilde{p}) \in \mathscr{D}_{\beta_{1}}^{l}(\Omega)$.

Рассмотрим последний случай: $\beta+\delta_{0} \in(-2,-1)$. Поскольку $(\mathbf{v} \cdot \nabla) \mathbf{v} \in$ $\mathscr{V}_{\left(\beta+\delta_{0}\right)+2,1}^{1}(\Omega)$, то $(\mathbf{v}, p)$ можно рассматривать как решение задачи Стокса $(2.23)$ с правой частью $(\mathbf{f}-(\mathbf{v} \cdot \nabla) \mathbf{v}, 0, \mathbf{h}) \in \mathscr{R}_{\beta+\delta_{0}}^{2}(\Omega ; \partial \Omega)$. В силу теоремы 2.1 (i) $(\mathbf{v}, p) \in \mathscr{D}_{\beta+\delta_{0}}^{2}(\Omega)$. Отсюда следует, что $(\mathbf{v} \cdot \nabla) \mathbf{v} \in \mathcal{V}_{\left(\beta+3 \delta_{0}\right)+2,1}^{1}(\Omega)$. Если $\beta+3 \delta_{0}<-1$, то продолжим этот процесс, пока не получим включение $(\mathbf{v} \cdot \nabla) \mathbf{v} \in$ $\mathscr{V}_{\left(\beta+2^{m} \delta_{0}-\delta_{0}\right)+2,1}^{1}(\Omega)$ с $\beta+\left(2^{m}-1\right) \delta_{0} \geqslant-1$. Если это неравенство строгое, то по доказанному $\mathbf{v}$ и $p$ допускают асимптотические представления (3.19), (3.20) с $(\widetilde{\mathbf{v}}, \tilde{p}) \in \mathscr{D}_{\beta_{1}}^{l}(\Omega)$. Если же $\beta+\left(2^{m}-1\right) \delta_{0}=1$, то $(\mathbf{f}-(\mathbf{v} \cdot \nabla) \mathbf{v}, 0, \mathbf{h}) \in$ $\mathscr{R}_{\beta+\left(2^{m}-1\right) \delta_{0}-\varepsilon}^{2}(\Omega ; \partial \Omega)$ и $(\mathbf{v}, p) \in \mathscr{D}_{\beta+\left(2^{m}-1\right) \delta_{0}-\varepsilon}^{2}(\Omega)$ с любым $\varepsilon>0$. Отсюда заключаем, что $(\mathbf{v} \cdot \nabla) \mathbf{v} \in \mathscr{V}_{\beta+\left(2^{m+1}-1\right) \delta_{0}+2 \varepsilon}(\Omega)$. Выберем $\varepsilon$ так, чтобы вьполнялось неравенство $\beta+\left(2^{m+1}-1\right) \delta_{0}+2 \varepsilon>-1$. Тогда опять приходим к случаю b) и, следовательно, справедливы представления (3.19), (3.20) с $(\widetilde{\mathbf{v}}, \widetilde{p}) \in \mathscr{D}_{\beta_{1}}^{l}(\Omega)$. Теорема доказана. 
ЗАмЕчАНИЕ 3.1. Из доказательства теоремы 3.2 следует, что если $(\mathbf{f}, 0, \mathbf{h}) \in$ $\mathscr{R}_{\beta_{1}}^{l}(\Omega ; \partial \Omega), \mathbf{v}^{\prime} \in \mathscr{V}_{\beta+1,2}^{3}(\Omega), v_{3} \in \mathscr{V}_{\beta+2,1}^{3}(\Omega)$ с $-2<\beta \leqslant \beta_{1}<-1, l \geqslant 2$, то $(\widetilde{\mathbf{v}}, \widetilde{p}) \in \mathscr{D}_{\beta_{1}}^{l}(\Omega)$.

ЗАмЕчАнИЕ 3.2. Утверждение теоремы остается в силе, если $\mathbf{v}^{\prime} \in \mathscr{V}_{\beta+1,1}^{2}(\Omega)$, $v_{3} \in \mathscr{V}_{\beta+2,0}^{2}(\Omega)$ с $\beta \in(-5 / 3,-1)$. При доказательстве этого факта надо лишш вместо неравенства (3.14) воспользоваться неравенством (3.15).

ЗАмечАниЕ 3.3. Теорема 3.2 носит условный характер. В $\S 4$ будет показано, что условия теоремы 3.2 выполнены для обобщенных решений задачи (3.1), имеющих ненулевой поток через сечения слоя П.

\section{§4. Решения с ненулевым потоком}

Сушествование обобщенных решений системы уравнений Навье-Стокса (3.1), имеющих ненулевой поток через сечения $S_{t}$ слоя П, было доказано в [17] (см. также [7], [11], где аналогичные результаты получены для областей $\Omega$, имеющих несколько выходов на бесконечность, один из которых совпадает со слоем П). Сформулируем результат, доказанньй в [17].

ТЕорема 4.1. Предположим, ито $\mathbf{f} \in L_{\mathrm{loc}}^{2}(\Omega) u$

$$
\begin{gathered}
\sup _{k \geqslant 0}\left\|\mathbf{f} ; L^{2}\left(\omega_{k}\right)\right\|<\infty, \quad \mathbf{h} \in W^{1 / 2,2}(\partial \Omega), \\
\operatorname{supp} \mathbf{h} \subset \partial \Omega \cap \bar{B}_{R_{0}}, \quad \int_{\partial \Omega} \mathbf{h} \cdot \mathbf{n} d s=F \neq 0 .
\end{gathered}
$$

Тогда существует обобщенное решение $\mathbf{v}$ задачи (3.1), удовлетворяющее условию

и неравенствам

$$
\int_{S_{t}} \mathbf{v} \cdot n^{\prime} d s=-F
$$

$$
\begin{aligned}
\int_{\Omega_{k}}|\nabla \mathbf{v}|^{2} d x \leqslant c k & \left(\sup _{l \geqslant 0}\left(l^{-1}\left\|\mathbf{f} ; L^{2}\left(\Omega_{l}\right)\right\|^{2}\right)+\left\|\mathbf{h} ; W^{1 / 2,2}(\partial \Omega)\right\|^{2}\right. \\
& \left.+\left\|\mathbf{h} ; W^{1 / 2,2}(\partial \Omega)\right\|^{4}\right) \equiv c_{1}(d) k, \quad k \geqslant 0 \\
\int_{\omega_{k}}|\nabla \mathbf{v}|^{2} d x \leqslant c\left(\sup _{l \geqslant 0}\left\|\mathbf{f} ; L^{2}\left(\omega_{l}\right)\right\|^{2}+\left\|\mathbf{h} ; W^{1 / 2,2}(\partial \Omega)\right\|^{2}\right. & \\
& \left.+\left\|\mathbf{h} ; W^{1 / 2,2}(\partial \Omega)\right\|^{4}\right) \equiv c_{2}(d), \quad k \geqslant 0
\end{aligned}
$$

Постоянные в (4.3), (4.4) не зависят от $k$.

Отметим, что в теореме 4.1 не предполагается малость данных задачи и потока $F$. В дальнейшем все постоянные, зависящие от данных задачи, будем обозначать через $c_{i}(d)$, не указывая их зависимость от различных норм $\mathbf{f}, \mathbf{h}$ и от $|F|$.

В этом параграфе будет доказано, что решение $\mathbf{v}$, гарантированное теоремой 4.1, ведет себя на бесконечности так же, как решение линейной задачи Стокса (2.23), т.е. что главные члены асимптотических представлений линейной и нелинейной задач совпадают. Для малых данных этот результат получен в [17] (см. также [7]). Доказательство основано на применении техники оценок типа Сент-Венана, развитой в [5], [10]-[12], и результатов $§ 3$. Нам потребуется следующая 
ЛЕмма 4.1. Предположим, что

$$
\begin{gathered}
y_{k+1} \geqslant y_{k}>0 \quad \forall k \geqslant 1 \\
\lim _{k \rightarrow \infty} k^{-3} y_{k}=0 .
\end{gathered}
$$

Если әлементы последовательности $\left\{y_{k}\right\}$ удовлетворяют неравенству

$$
y_{k} \leqslant b\left(y_{k+1}-y_{k}\right)+a\left(y_{k+1}-y_{k}\right)^{3 / 2}+c_{0},
$$

әде $a>0, b>0, c_{0}>0$, то справедлива оценка

$$
y_{k} \leqslant c_{0} \quad \forall k \geqslant 1 \text {. }
$$

ДокАЗАтЕльство. Утверждение, содержащее как частный случай лемму 4.1, доказано в работе [10]. Однако для удобства читателя докажем лемму 4.1 непосредственно. Положим $z_{k}=y_{k}-c_{0}$ и перепишем (4.5) в виде

$$
z_{k} \leqslant b\left(z_{k+1}-z_{k}\right)+a\left(z_{k+1}-z_{k}\right)^{3 / 2} .
$$

Предположим, что оценка (4.6) неверна. Тогда найдется $k_{0}$ такое, что $z_{k_{0}}>0$. Из неравенства (4.7) при $k=k_{0}$ следует, что $z_{k_{0}+1}-z_{k_{0}} \geqslant \alpha_{0}>0$ для некоторого $\alpha_{0}$. Так как $z_{k+1} \geqslant z_{k}$, то

$$
z_{k+1} \geqslant z_{k}+\alpha_{0} \quad \forall k \geqslant k_{0}
$$

Отсюда по индукции выводим неравенство

$$
z_{k_{0}+l} \geqslant l \alpha_{0}+z_{k_{0}}
$$

и, следовательно, $\lim _{l \rightarrow \infty} z_{k_{0}+l}=\infty$. Теперь из (4.7) вытекает соотношение

$$
\lim _{l \rightarrow \infty}\left(z_{k_{0}+l+1}-z_{k_{0}+l}\right)=\infty .
$$

Поэтому для достаточно больших $l$

$$
z_{k_{0}+l+1}-z_{k_{0}+l}>1
$$

Без ограничения общности можно считать, что последнее неравенство верно для всех $l \geqslant 0$. Из (4.7) следует неравенство

$$
z_{k_{0}+l} \leqslant(a+b)\left(z_{k_{0}+l+1}-z_{k_{0}+l}\right)^{3 / 2} .
$$

Отсюда выводим

$$
z_{k_{0}+l+1} \geqslant z_{k_{0}+l}+\nu_{0} z_{k_{0}+l}^{2 / 3}, \quad \nu_{0}=(a+b)^{-2 / 3} .
$$

При помощи индукции легко показать, что (4.8) влечет оценку

$$
z_{k_{0}+l+1} \geqslant \nu_{1} l^{3}, \quad \nu_{1}=\left(\frac{\nu_{0}}{9}\right)^{3}
$$

которая противоречит условию $\lim _{k \rightarrow \infty} k^{-3} z_{l}=0$. Таким образом, $z_{k} \leqslant 0 \forall k \geqslant 1$ и справедлива оценка (4.6). 
ЛЕмма 4.2. Пусть $\mathbf{v}$ - обобщенное решение задачи (3.1), удовлетворяющее неравенству (4.4). Тогда $\nabla \mathbf{v} \in L_{-\varepsilon}^{2}(\Omega) u$

$$
\int_{\Omega}\left(1+r^{2}\right)^{-\varepsilon}|\nabla \mathbf{v}|^{2} d x \leqslant c_{3}(d) \quad \forall \varepsilon>0 .
$$

ДоказАтЕльство. Имеем (см. (4.4))

$$
\begin{aligned}
& \int_{\Omega_{l}}\left(1+r^{2}\right)^{-\varepsilon}|\nabla \mathbf{v}|^{2} d x \leqslant c \sum_{j=0}^{l} \int_{\omega_{j}}\left(1+r^{2}\right)^{-\varepsilon}|\nabla \mathbf{v}|^{2} d x \\
& \leqslant c \sum_{j=0}^{\infty} 2^{-2 j \varepsilon} \int_{\omega_{j}}|\nabla \mathbf{v}|^{2} d x \leqslant c_{2}(d) \sum_{j=0}^{\infty}\left(2^{-2 \varepsilon}\right)^{j} \leqslant c_{3}(d)
\end{aligned}
$$

Переходя к пределу при $l \rightarrow \infty$, получаем утверждение леммы.

Лемма 4.3. Пусть $\mathbf{f} \in L^{2}(\Omega), \mathbf{h} \in W^{3 / 2,2}(\partial \Omega)$. Тогда обобщенное решение $\mathbf{v}$ задачи (3.1) удовлетворяет включению $\mathbf{v} \in C^{1 / 2}(\bar{\Omega})$ и неравенству

$$
\left\|\mathbf{v} ; C^{1 / 2}(\bar{\Omega})\right\| \leqslant c_{4}(d)
$$

ДоказАтельство. Рассмотрим решение $\mathbf{v}$ в области $Q_{k, s}^{*}, k, s \in \mathbb{Z}$ (см. обозначения п. 2.2). Так как $Q_{k, s}$ - ограниченная область и $Q_{k, s} \subset Q_{k, s}^{*}$, то имеет место оценка (см. [22; гл. V, оценка (3.6)])

$$
\left\|\mathbf{v} ; C^{1 / 2}\left(\bar{Q}_{k, s}\right)\right\| \leqslant c\left\|\mathbf{v} ; W^{2,2}\left(Q_{k, s}\right)\right\| \leqslant c\left(\left\|\mathbf{v} ; W^{1,2}\left(Q_{k, s}^{*}\right)\right\|^{4}+\left\|\mathbf{f} ; L^{2}\left(Q_{k, s}^{*}\right)\right\|\right) .
$$

Поскольку для достаточно больших $k$ и $s$ области $Q_{k, s}$ отличаются лишь расположением в пространстве, то постоянная $c$ в последнем неравенстве может быть выбрана не зависяшей от $k, s \in \mathbb{Z}$. Пусть число $l=l(k, s)$ таково, что $\omega_{l}^{*} \supset Q_{k, s}^{*}$, где $\omega_{l}^{*}=\omega_{l-1} \cup \omega_{l} \cup \omega_{l+1}$. Тогда в силу (4.4) получим оценку

$$
\left\|\mathbf{v} ; C^{1 / 2}\left(\bar{Q}_{k, s}\right)\right\| \leqslant c\left(\left\|\mathbf{v} ; W^{1,2}\left(\omega_{l}^{*}\right)\right\|^{4}+\left\|\mathbf{f} ; L^{2}\left(\omega_{l}^{*}\right)\right\|\right) \leqslant c\left(c_{2}(d)^{4}+c_{5}(d)\right) \equiv c_{4}(d),
$$

где $c_{5}(d)$ зависит от норм $\left\|\mathbf{f} ; L^{2}(\Omega)\right\|$ и $\left\|\mathbf{h} ; W^{3 / 2,2}(\partial \Omega)\right\|$. Взяв в последнем неравенстве супремум по всем $k, z \in \mathbb{Z}$, выводим (4.10).

Лемма 4.4. Пусть $\mathbf{h} \in W^{l+1 / 2,2}(\partial \Omega), \operatorname{supp} \mathbf{h} \subset \partial \Omega \cup \bar{B}_{R_{0}}, \int_{\partial \Omega} \mathbf{h} \cdot \mathbf{n} d s=$ $F \neq 0$. Тогда в области $\Omega$ существует вектор-функиия $\mathbf{A}$, удовлетворяющая условиям

$$
\begin{gathered}
\left.\mathbf{A}\right|_{\partial \Omega}=\mathbf{h}, \quad \operatorname{div} \mathbf{A}=0, \quad \int_{S_{t}} \mathbf{A} \cdot \mathbf{n}^{\prime} d s=-F \\
\mathbf{A}(x)=-12 \nu F \mathbf{U}_{0}^{-}(y, z)=\frac{3 F}{\pi} z(z-1)\left(\frac{y}{r^{2}}, 0\right) \quad n p u \quad r \geqslant 3 R_{0} .
\end{gathered}
$$

Kроме того, $\mathbf{A} \in \mathscr{V}_{-\varepsilon, l+1}^{l+1}(\Omega) \forall \varepsilon>0 u$

$$
\left\|\mathbf{A} ; \mathscr{V}_{-\varepsilon, l+1}^{l+1}(\Omega)\right\| \leqslant c\left\|\mathbf{h} ; W^{l+1 / 2,2}(\partial \Omega)\right\| .
$$


ДокАЗАТЕЛЬСТво. Пусть $\mathbf{A}_{1} \in W^{l+1,2}(\Omega)$ - продолжение функции $\mathbf{h} \in$ $W^{l+1 / 2,2}(\partial \Omega)$ в $\Omega$. Поскольку supp $\mathbf{h} \subset \partial \Omega \cap B_{R_{0}}$, то $\mathbf{A}_{1}$ можно выбрать так, что supp $\mathbf{A}_{1} \subset \bar{\Omega}\left(2 R_{0}\right)$. Кроме того, имеет место оценка

$$
\left\|\mathbf{A}_{1} ; W^{l+1,2}(\Omega)\right\| \leqslant c\left\|\mathbf{h} ; W^{l+1 / 2,2}(\partial \Omega)\right\| .
$$

Рассмотрим в $\Omega$ задачу

$$
\begin{gathered}
\operatorname{div} \mathbf{A}_{2}=-\operatorname{div} \mathbf{A}_{1}+12 \nu F \nabla \chi \cdot \mathbf{U}_{0}^{-} \equiv g_{F}, \quad x \in \Omega, \\
\mathbf{A}_{2}=0, \quad x \in \partial \Omega,
\end{gathered}
$$

где $\chi$-срезающая функция из теоремы 3.2. Так как $\operatorname{supp} g_{F} \subset \Omega\left(2 R_{0}\right)$ и

$$
\begin{aligned}
\int_{\Omega\left(2 R_{0}\right)} g_{F} d x & =\int_{\Omega\left(2 R_{0}\right)}\left[-\operatorname{div} \mathbf{A}_{1}+12 \nu F \nabla \chi \cdot \mathbf{U}_{0}^{-}\right] d x \\
& =-\int_{\partial \Omega} \mathbf{h} \cdot \mathbf{n} d s+12 \nu F \int_{S_{2 R_{0}}} \mathbf{U}_{0}^{-} \cdot \mathbf{n}^{\prime} d s=-F+F=0,
\end{aligned}
$$

то согласно лемме 2.6 сушествует решение $\mathbf{A}_{2} \in W^{l+1,2}(\Omega)$ задачи (4.16) такое, что $\operatorname{supp} \mathbf{A}_{2} \subset \Omega\left(3 R_{0}\right)$ и

$$
\begin{aligned}
\left\|\mathbf{A}_{2} ; W^{l+1,2}(\Omega)\right\| & \leqslant c\left\|g_{F} ; W^{l, 2}(\Omega)\right\| \leqslant c\left(\left\|\mathbf{h} ; W^{l+1 / 2,2}(\partial \Omega)\right\|+|F|\right) \\
& \leqslant c\left\|\mathbf{h} ; W^{l+1 / 2,2}(\partial \Omega)\right\| .
\end{aligned}
$$

Положим $\mathbf{A}=\mathbf{A}_{1}+\mathbf{A}_{2}-12 \nu F \chi \mathbf{U}_{0}^{-}$. Очевидно, что $\operatorname{div} \mathbf{A}=0,\left.\mathbf{A}\right|_{\partial \Omega}=\mathbf{h}$ и вьполнено представление (4.13). Следовательно,

$$
\int_{S_{t}} \mathbf{A} \cdot \mathbf{n}^{\prime} d s=-12 \nu F \int_{S_{t}} \mathbf{U}_{0}^{-} \cdot \mathbf{n}^{\prime} d s=-F
$$

Из явной формулы для $\mathbf{U}_{0}^{-}$легко получить оценку

$$
\left\|12 \nu F \chi \mathbf{U}_{0}^{-} ; \mathscr{V}_{-\varepsilon, l+1}^{l+1}(\Omega)\right\| \leqslant c|F| \leqslant c\left\|\mathbf{h} ; W^{l+1 / 2,2}(\partial \Omega)\right\| .
$$

Так как функции $\mathbf{A}_{1}$ и $\mathbf{A}_{2}$ финитны, то нормы $\left\|\mathbf{A}_{i} ; W^{l+1,2}(\Omega)\right\|$ и $\left\|\mathbf{A}_{i} ; \mathcal{V}_{-\varepsilon, l+1}^{l+1}(\Omega)\right\|$, $i=1,2$, эквивалентны, и требуемое неравенство (4.14) следует из $(4.15),(4.17)$, (4.18).

Вернемся к рассмотрению задачи (3.1). Пусть $\mathbf{f} \in \mathcal{V}_{\beta_{1}+2, l-1}^{l-1}(\Omega), l \geqslant 1, \beta_{1} \in$ $(-1,0), \mathbf{h} \in W^{l+1 / 2,2}(\partial \Omega)$. Тогда обобщенное решение $\mathbf{v} \in W_{\text {loc }}^{l+1,2}(\Omega)$ и существует функция $p \in W_{\mathrm{loc}}^{l, 2}(\Omega)$ такая, что $(\mathbf{v}, p)$ удовлетворяет системе (3.1) почти всюду в $\Omega$. Сделаем в (3.1) замену

$$
\mathbf{u}=\mathbf{v}-\mathbf{A}, \quad q=p+12 \nu F \chi(r) P_{0}^{-}(y) .
$$


Для $(\mathbf{u}, q)$ получим задачу

$$
\begin{gathered}
-\nu \Delta \mathbf{u}+\nabla q=\mathbf{f}_{1}-[(\mathbf{A} \cdot \nabla) \mathbf{u}+(\mathbf{u} \cdot \nabla) \mathbf{A}+(\mathbf{u} \cdot \nabla) \mathbf{u}], \quad x \in \Omega, \\
\operatorname{div} \mathbf{u}=0, \quad x \in \Omega, \\
\mathbf{u}=0, \quad x \in \partial \Omega, \\
\int_{S_{t}} \mathbf{u} \cdot \mathbf{n}^{\prime} d s=0,
\end{gathered}
$$

где $\mathbf{f}_{1}=\mathbf{f}+\left(\nu \Delta \mathbf{A}+12 \nu F \nabla\left(\chi P_{0}^{-}\right)\right)-(\mathbf{A} \cdot \nabla) \mathbf{A}$. Поскольку $\left(\mathbf{U}_{0}^{-}, P_{0}^{-}\right)$удовлетворяет системе Стокса, то функция $\nu \Delta \mathbf{A}+12 \nu F \nabla\left(\chi P_{0}^{-}\right)$имеет компактный носитель, содержащийся в $\Omega\left(3 R_{0}\right)$. Кроме того, используя структуру $\mathbf{U}_{0}^{-}$, легко показать, что при $r \geqslant 3 R_{0}$ третья компонента вектор-функции $(\mathbf{A} \cdot \nabla) \mathbf{A}$ тождественно равна нулю и

$$
\left|\partial_{y}^{\gamma} \partial_{z}^{\alpha} \mathbf{A}(x)\right| \leqslant c|F|\left(1+r^{2}\right)^{-(1+|\gamma|) / 2} \quad \text { при } r \geqslant 3 R_{0} .
$$

Поэтому $(\mathbf{A} \cdot \nabla) \mathbf{A} \in \mathcal{V}_{\beta+2, l-1}^{l-1}(\Omega) \forall \beta<0$ и

$$
\begin{aligned}
\left\|(\mathbf{A} \cdot \nabla) \mathbf{A} ; \mathscr{V}_{\beta+2, l-1}^{l-1}(\Omega)\right\| & \leqslant c\left(\left\|\mathbf{h} ; W^{l+1 / 2,2}(\partial \Omega)\right\|^{2}+|F|\right) \\
& \leqslant c\left\|\mathbf{h} ; W^{l+1 / 2,2}(\partial \Omega)\right\|^{2}
\end{aligned}
$$

Таким образом, $\mathbf{f}_{1} \in \mathscr{V}_{\beta_{1}+2, l-1}^{l-1}(\Omega)$,

$$
\begin{gathered}
\left\|\mathbf{f}_{1} ; \mathscr{V}_{\beta_{1}+2, l-1}^{l-1}(\Omega)\right\| \leqslant \\
c\left(\left\|\mathbf{h} ; W^{l+1 / 2,2}(\partial \Omega)\right\|+\left\|\mathbf{h} ; W^{l+1 / 2,2}(\partial \Omega)\right\|^{2}\right. \\
\left.+\left\|\mathbf{f} ; \mathscr{V}_{\beta_{1}+2, l-1}^{l-1}(\Omega)\right\|\right) .
\end{gathered}
$$

Лемма 4.5. Пусть $\mathbf{f} \in L_{\beta_{1}+2}^{2}(\Omega), \beta_{1} \in(-1,0), \mathbf{h} \in W^{3 / 2,2}(\partial \Omega)$. Тогда $\nabla \mathbf{u} \in L^{2}(\Omega)$ и имеет место оценка

$$
\int_{\Omega}|\nabla \mathbf{u}|^{2} d x \leqslant c_{6}(d)
$$

ДокАЗАТЕЛЬство. Легко видеть, что и удовлетворяет интегральному тождеству

$$
\nu \int_{\Omega} \nabla \mathbf{u} \cdot \nabla \boldsymbol{\eta} d x=\int_{\Omega} \mathbf{f}_{1} \cdot \boldsymbol{\eta} d x-\int_{\Omega}((\mathbf{A} \cdot \nabla) \mathbf{u}+(\mathbf{u} \cdot \nabla) \mathbf{A}+(\mathbf{u} \cdot \nabla) \mathbf{u}) \cdot \boldsymbol{\eta} d x
$$

для любой вектор-функции $\boldsymbol{\eta} \in W_{\mathrm{loc}}^{1,2}(\Omega)$ такой, что $\operatorname{div} \boldsymbol{\eta}=0,\left.\boldsymbol{\eta}\right|_{\partial \Omega}=0$ и $\boldsymbol{\eta}(x)=0$ при $r \gg 1$.

В области $\Omega$ определим функцию

$$
\mathbf{U}_{k}= \begin{cases}\mathbf{u}, & x \in \Omega_{k}, \\ \xi_{k} \mathbf{u}+\mathbf{w}_{k}, & x \in \omega_{k}, \\ 0, & x \in \Omega \backslash \Omega_{k+1},\end{cases}
$$


где $\mathbf{w}_{k}-$ решение задачи

$$
\begin{gathered}
\operatorname{div} \mathbf{w}_{k}=-\operatorname{div}\left(\xi_{k} \mathbf{u}\right)=-\nabla \xi_{k} \cdot \mathbf{u}, \quad x \in \omega_{k}, \\
\mathbf{w}_{k}=0, \quad x \in \partial \omega_{k},
\end{gathered}
$$

а $\xi_{k}$ - гладкая срезающая функция, $\xi_{k}(r)=1$ при $x \in \Omega_{k}, \xi_{k}(r)=0$ при $x \in$ $\Omega \backslash \Omega_{k+1}$ и

$$
\left|\nabla \xi_{k}\right| \leqslant c 2^{-k}
$$

Так как

$$
\int_{\omega_{k}} \operatorname{div}\left(\xi_{k} \mathbf{u}\right) d x=-\int_{S_{2^{k}}} \xi_{k}(r) \mathbf{u} \cdot \mathbf{n}^{\prime} d s=-\int_{S_{2^{k}}} \mathbf{u} \cdot \mathbf{n}^{\prime} d s=0
$$

то в силу леммы 2.7 существует решение задачи (4.23), удовлетворяющее неравенству

$$
\left\|\nabla \mathbf{w}_{k} ; L^{2}\left(\omega_{k}\right)\right\| \leqslant c 2^{k}\left\|\nabla \xi_{k} \cdot \mathbf{u} ; L^{2}\left(\omega_{k}\right)\right\| \leqslant c\left\|\mathbf{u} ; L^{2}\left(\omega_{k}\right)\right\| \leqslant c\left\|\nabla \mathbf{u} ; L^{2}\left(\omega_{k}\right)\right\|
$$

в котором постоянная $c$ не зависит от $k$.

Легко видеть, что $\operatorname{div} \mathbf{U}_{k}=0,\left.\mathbf{U}_{k}\right|_{\partial \Omega}=0$ и $\operatorname{supp} \mathbf{U}_{k} \subset \Omega_{k+1}$. Взяв в интегральном тождестве (4.22) $\boldsymbol{\eta}=\mathbf{U}_{k}$, получим соотношение

$\nu \int_{\Omega_{k+1}} \nabla \mathbf{u} \cdot \nabla \mathbf{U}_{k} d x=\int_{\Omega_{k+1}} \mathbf{f}_{1} \cdot \mathbf{U}_{k} d x-\int_{\Omega_{k+1}}[(\mathbf{A} \cdot \nabla) \mathbf{u}+(\mathbf{u} \cdot \nabla) \mathbf{A}+(\mathbf{u} \cdot \nabla) \mathbf{u}] \cdot \mathbf{U}_{k} d x$.

Так как

$$
\int_{\Omega_{k+1}} \nabla \mathbf{u} \cdot \nabla \mathbf{U}_{k} d x=\int_{\Omega_{k}}|\nabla \mathbf{u}|^{2} d x+\int_{\omega_{k}} \nabla \mathbf{u} \cdot \nabla\left(\xi_{k} \mathbf{u}+\mathbf{w}_{k}\right) d x
$$

то последнее равенство можно переписать в виде

$$
\begin{aligned}
& \nu \int_{\Omega_{k}}|\nabla \mathbf{u}|^{2} d x=-\nu \int_{\omega_{k}} \nabla \mathbf{u} \cdot \nabla\left(\xi_{k} \mathbf{u}+\mathbf{w}_{k}\right) d x \\
& \quad-\int_{\Omega_{k+1}}((\mathbf{A} \cdot \nabla) \mathbf{u}+(\mathbf{u} \cdot \nabla) \mathbf{A}+(\mathbf{u} \cdot \nabla) \mathbf{u}) \cdot \mathbf{U}_{k} d x \\
& \quad+\int_{\Omega_{k+1}} \mathbf{f}_{1} \cdot \mathbf{U}_{k} d x=J_{1}+J_{2}+J_{3} .
\end{aligned}
$$

Оценим правую часть (4.26). Рассмотрим слагаемое $J_{1}$. В силу неравенств Гёльдера, Фридрихса и $(4.24),(4.25)$ имеем

$$
\begin{aligned}
\left|J_{1}\right| \leqslant & \nu\left|\int_{\omega_{k}} \nabla \mathbf{u} \cdot \nabla\left(\xi_{k} \mathbf{u}+\mathbf{w}_{k}\right) d x\right| \leqslant \nu\left(\int_{\omega_{k}}|\nabla \mathbf{u}|^{2} d x\right)^{1 / 2} \\
& \times\left(\int_{\omega_{k}}\left(\left|\nabla \xi_{k}\right|^{2}|\mathbf{u}|^{2}+\left|\xi_{k}\right|^{2}|\nabla \mathbf{u}|^{2}+\left|\nabla \mathbf{w}_{k}\right|^{2}\right) d x\right)^{1 / 2} \\
\leqslant & c\left(\int_{\omega_{k}}|\nabla \mathbf{u}|^{2} d x\right)^{1 / 2}\left(\int_{\omega_{k}}\left(2^{-2 k}|\mathbf{u}|^{2}+|\nabla \mathbf{u}|^{2}+\left|\nabla \mathbf{w}_{k}\right|^{2}\right) d x\right)^{1 / 2} \\
\leqslant & c \int_{\omega_{k}}|\nabla \mathbf{u}|^{2} d x .
\end{aligned}
$$


Второе слагаемое в правой части (4.26) представим в виде суммы

$$
J_{2}=\int_{\Omega_{k+1}}((\mathbf{A} \cdot \nabla) \mathbf{u}+(\mathbf{u} \cdot \nabla) \mathbf{A}) \cdot \mathbf{U}_{k} d x+\int_{\Omega_{k+1}}(\mathbf{u} \cdot \nabla) \mathbf{u} \cdot \mathbf{U}_{k} d x \equiv J_{21}+J_{22}
$$

Для оценки $J_{21}$ заметим, что

$$
\int_{\Omega_{k+1}}((\mathbf{A} \cdot \nabla) \mathbf{u}+(\mathbf{u} \cdot \nabla) \mathbf{A}) \cdot \mathbf{U}_{k} d x=-\int_{\Omega_{k+1}}\left((\mathbf{A} \cdot \nabla) \mathbf{U}_{k} \cdot \mathbf{u}+(\mathbf{u} \cdot \nabla) \mathbf{U}_{k} \cdot \mathbf{A}\right) d x
$$

и воспользуемся неравенствами Юнга и Фридрихса:

$$
\left|J_{21}\right| \leqslant c_{\delta} \int_{\Omega_{k+1}}|\mathbf{A}|^{2}|\mathbf{u}|^{2} d x+\delta \int_{\Omega_{k+1}}\left|\nabla \mathbf{U}_{k}\right|^{2} d x
$$

Поскольку $\mathbf{A} \in W^{2,2}\left(\Omega\left(3 R_{0}\right)\right)$ (см. доказательство леммы 4.4$)$, то

$$
\sup _{x \in \overline{\Omega\left(3 R_{0}\right)}}|\mathbf{A}(x)|^{2} \leqslant c\left\|\mathbf{A} ; W^{2,2}\left(\Omega\left(3 R_{0}\right)\right)\right\|^{2} \leqslant c\left\|\mathbf{h} ; W^{3 / 2,2}(\partial \Omega)\right\|^{2} .
$$

Далее, так как $\mathbf{A}(x)=-12 \nu F \mathbf{U}_{0}^{-}(y, z)$ при $r \geqslant 3 R_{0}$, то

$$
|\mathbf{A}(x)|^{2} \leqslant c|F|^{2}\left(1+r^{2}\right)^{-1} \leqslant c\left(1+r^{2}\right)^{-1}\left\|\mathbf{h} ; W^{3 / 2,2}(\partial \Omega)\right\|^{2} \text { при } r \geqslant 3 R_{0} .
$$

Используя оценки (4.9), (4.14), отсюда выводим

$$
\begin{aligned}
\int_{\Omega_{k+1}}|\mathbf{A}|^{2}|\mathbf{u}|^{2} d x & \leqslant c\left\|\mathbf{h} ; W^{3 / 2,2}(\partial \Omega)\right\|^{2} \int_{\Omega_{k+1}}\left(1+r^{2}\right)^{-1}|\mathbf{u}|^{2} d x \\
& \leqslant c\left\|\mathbf{h} ; W^{3 / 2,2}(\partial \Omega)\right\|^{2} \int_{\Omega_{k+1}}\left(1+r^{2}\right)^{-1}\left(|\mathbf{v}|^{2}+|\mathbf{A}|^{2}\right) d x \\
& \leqslant c\left\|\mathbf{h} ; W^{3 / 2,2}(\partial \Omega)\right\|^{2} \int_{\Omega}\left(1+r^{2}\right)^{-1}\left(|\nabla \mathbf{v}|^{2}+|\mathbf{A}|^{2}\right) d x \leqslant c_{7}(d) .
\end{aligned}
$$

Таким образом,

$$
\begin{aligned}
J_{21} & \leqslant c_{\delta} c_{7}(d)+\delta \int_{\Omega_{k+1}}\left|\nabla \mathbf{U}_{k}\right|^{2} d x \\
& \leqslant c_{\delta} c_{7}(d)+\delta \int_{\Omega_{k}}|\nabla \mathbf{u}|^{2} d x+\delta \int_{\omega_{k}}\left|\nabla\left(\xi_{k} \mathbf{u}+\mathbf{w}_{k}\right)\right|^{2} d x \\
& \leqslant c_{\delta} c_{7}(d)+\delta \int_{\Omega_{k}}|\nabla \mathbf{u}|^{2} d x+c \delta \int_{\omega_{k}}|\nabla \mathbf{u}|^{2} d x .
\end{aligned}
$$

Рассмотрим слагаемое $J_{22}$. Так как $\operatorname{supp}_{k} \subset \omega_{k}$, то

$$
J_{22}=\int_{\Omega_{k+1}}(\mathbf{u} \cdot \nabla) \mathbf{u} \cdot \mathbf{U}_{k} d x=\int_{\Omega_{k+1}}(\mathbf{u} \cdot \nabla) \mathbf{u} \cdot \xi_{k} \mathbf{u} d x+\int_{\omega_{k}}(\mathbf{u} \cdot \nabla) \mathbf{u} \cdot \mathbf{w}_{k} d x
$$


Используя соотношения $\xi_{k}(r)=0$ при $r \geqslant 2^{k+1}, \operatorname{supp} \nabla \xi_{k} \subset \omega_{k}$, и интегрируя по частям, находим

$$
\int_{\Omega_{k+1}}(\mathbf{u} \cdot \nabla) \mathbf{u} \cdot \xi_{k} \mathbf{u} d x=-\frac{1}{2} \int_{\omega_{k}} \mathbf{u} \cdot \nabla \xi_{k}|\mathbf{u}|^{2} d x
$$

Далее, поскольку $\left.\mathbf{w}_{k}\right|_{\partial \omega_{k}}=0$, то

$$
\int_{\omega_{k}}(\mathbf{u} \cdot \nabla) \mathbf{u} \cdot \mathbf{w}_{k} d x=-\int_{\omega_{k}}(\mathbf{u} \cdot \nabla) \mathbf{w}_{k} \cdot \mathbf{u} d x
$$

Поэтому

$$
\begin{aligned}
\left|J_{22}\right| & \leqslant \frac{1}{2} \int_{\omega_{k}}\left|\nabla \xi_{k}\right||\mathbf{u}|^{3} d x+\left(\int_{\omega_{k}}|\mathbf{u}|^{4} d x\right)^{1 / 2}\left(\int_{\omega_{k}}\left|\nabla \mathbf{w}_{k}\right|^{2} d x\right)^{1 / 2} \\
& \leqslant c \int_{\omega_{k}}|\mathbf{u}|^{3} d x+c\left(\int_{\omega_{k}}|\mathbf{u}|^{4} d x\right)^{1 / 2}\left(\int_{\omega_{k}}|\nabla \mathbf{u}|^{2} d x\right)^{1 / 2} .
\end{aligned}
$$

В силу леммы 2.1 справедлива оценка

$$
\left\|\mathbf{u} ; L^{3}\left(\omega_{k}\right)\right\|+\left\|\mathbf{u} ; L^{4}\left(\omega_{k}\right)\right\| \leqslant c\left\|\mathbf{u} ; W^{1,2}\left(\omega_{k}\right)\right\| \leqslant c\left\|\nabla \mathbf{u} ; L^{2}\left(\omega_{k}\right)\right\|
$$

где постоянная не зависит от $k$. Это дает

$$
\left|J_{22}\right| \leqslant c\left(\int_{\omega_{k}}|\nabla \mathbf{u}|^{2} d x\right)^{3 / 2}
$$

и из $(4.28),(4.29)$ следует, что

$$
\left|J_{2}\right| \leqslant c_{\delta} c_{7}(d)+c \delta \int_{\Omega_{k}}|\nabla \mathbf{u}|^{2} d x+c \delta \int_{\omega_{k}}|\nabla \mathbf{u}|^{2} d x+c\left(\int_{\omega_{k}}|\nabla \mathbf{u}|^{2} d x\right)^{3 / 2}
$$

Наконец, для слагаемого $J_{3}$ справедлива оценка

$$
\begin{aligned}
\left|J_{3}\right| & =\left|\int_{\Omega_{k+1}} \mathbf{f}_{1} \cdot \mathbf{U}_{k} d x\right| \leqslant c_{\delta} \int_{\Omega_{k+1}}\left|\mathbf{f}_{1}\right|^{2} d x+\delta \int_{\Omega_{k+1}}\left|\mathbf{U}_{k}\right|^{2} d x \\
& \leqslant c_{\delta} \int_{\Omega}\left|\mathbf{f}_{1}\right|^{2} d x+\delta \int_{\Omega_{k}}|\nabla \mathbf{u}|^{2} d x+\delta \int_{\omega_{k}}\left|\nabla\left(\xi_{k} \mathbf{u}+\mathbf{w}_{k}\right)\right|^{2} d x \\
& \leqslant c_{8}(d)+\delta \int_{\Omega_{k}}|\nabla \mathbf{u}|^{2} d x+c \delta \int_{\omega_{k}}|\nabla \mathbf{u}|^{2} d x
\end{aligned}
$$

Собирая неравенства $(4.27),(4.30),(4.31)$, из (4.26) вьводим соотношение

$$
\begin{aligned}
\nu \int_{\Omega_{k}}|\nabla \mathbf{u}|^{2} d x \leqslant & c_{1} \delta \int_{\Omega_{k}}|\nabla \mathbf{u}|^{2} d x+c_{2} \int_{\omega_{k}}|\nabla \mathbf{u}|^{2} d x \\
& +c_{3}\left(\int_{\omega_{k}}|\nabla \mathbf{u}|^{2} d x\right)^{3 / 2}+c_{9}(d) .
\end{aligned}
$$


Выберем и зафиксируем $\delta$ так, чтобы выполнялось неравенство $c_{1} \delta<\nu / 2$. Тогда из (4.32) следует, что

$$
\int_{\Omega_{k}}|\nabla \mathbf{u}|^{2} d x \leqslant c_{4} \int_{\omega_{k}}|\nabla \mathbf{u}|^{2} d x+c_{5}\left(\int_{\omega_{k}}|\nabla \mathbf{u}|^{2} d x\right)^{3 / 2}+2 \nu^{-1} c_{9}(d) .
$$

Положим $y_{k}=\int_{\Omega_{k}}|\nabla \mathbf{u}|^{2} d x$. Так как

$$
y_{k+1}=\int_{\Omega_{k+1}}|\nabla \mathbf{u}|^{2} d x=y_{k}+\int_{\omega_{k}}|\nabla \mathbf{u}|^{2} d x
$$

то неравенство (4.33) можно переписать в виде

$$
y_{k} \leqslant c_{4}\left(y_{k+1}-y_{k}\right)+c_{5}\left(y_{k+1}-y_{k}\right)^{3 / 2}+2 \nu^{-1} c_{9}(d)
$$

В силу оценки (4.3)

$$
y_{k} \leqslant c_{1}(d) k
$$

Следовательно, $\lim _{k \rightarrow \infty} k^{-3} y_{k}=0$ и согласно лемме 4.1 из (4.34) следует, что

$$
y_{k}=\int_{\Omega_{k}}|\nabla \mathbf{u}|^{2} d x \leqslant 2 \nu^{-1} c_{9}(d) .
$$

Переходя в $(4.35)$ к пределу при $k \rightarrow \infty$, получаем неравенство

$$
\int_{\Omega}|\nabla \mathbf{u}|^{2} d x \leqslant 2 \nu^{-1} c_{9}(d)
$$

Лемма доказана.

Лемма 4.6. Пусть выполнены условия леммы 4.5. Тогда существует такое число $\beta_{*}=\beta_{*}(d) \in(0,1 / 2)$, что $\nabla \mathbf{u} \in L_{\mu}^{2}(\Omega) \forall \mu \in\left(0, \beta_{*}\right) u$

$$
\int_{\Omega}\left(1+r^{2}\right)^{\mu}|\nabla \mathbf{u}|^{2} d x \leqslant c_{10}(d)
$$

ДокАЗАТЕЛьство. Из мультипликативного неравенства (см., например, [22])

$$
\left\|\mathbf{u} ; L^{q}(\Omega)\right\| \leqslant(48)^{\alpha / 6}\left\|\nabla \mathbf{u} ; L^{2}(\Omega)\right\|^{\alpha}\left\|\mathbf{u} ; L^{2}(\Omega)\right\|^{1-\alpha} \leqslant c\left\|\nabla \mathbf{u} ; L^{2}(\Omega)\right\|,
$$

справедливого для $q \in[2,6]$ и $\alpha=3 / 2-3 / q$, находим, что $\mathbf{u} \in L^{4}(\Omega)$. Поэтому интегральное тождество (4.22) можно распространить по непрерьвности на соленоидальные вектор-функции $\boldsymbol{\eta}$ с конечным интегралом Дирихле $\int_{\Omega}|\nabla \boldsymbol{\eta}|^{2} d x$.

Пусть $k \geqslant 1$. Определим в области $\Omega$ непрерывную функцию

$$
R_{\mu}(x, k)= \begin{cases}5^{\mu}, & r \leqslant 2 \\ \left(1+r^{2}\right)^{\mu}, & 2 \leqslant r \leqslant 2^{k} \\ \left(1+2^{2 k}\right)^{\mu}, & r \geqslant 2^{k}\end{cases}
$$


Легко видеть, что $\operatorname{supp}\left(\nabla R_{\mu}(x, k)\right) \subset \Omega_{k} \backslash \omega_{0}$ и

$$
R_{\mu}(x, k) \leqslant c\left(1+r^{2}\right), \quad\left|\nabla R_{\mu}(x, k)\right| \leqslant c \mu\left(1+r^{2}\right)^{\mu-1 / 2} \quad \forall x \in \Omega, \quad k \geqslant 1
$$

причем постоянные в (4.37) не зависят от $x$ и $k$. Положим в интегральном тождестве $(4.22)$

$$
\eta(x)= \begin{cases}R_{\mu}(x, k) \mathbf{u}(x), & x \in \omega_{0}, \\ R_{\mu}(x, k) \mathbf{u}+\mathbf{W}_{j}(x), & x \in \omega_{j}, j=1, \ldots, k-1, \\ R_{\mu}(x, k) \mathbf{u}(x), & x \in \Omega \backslash \Omega_{k},\end{cases}
$$

где функции $\mathbf{W}_{j}$ являются решениями задач

$$
\begin{gathered}
\operatorname{div} \mathbf{W}_{j}=-\operatorname{div}\left(R_{\mu}(x, k) \mathbf{u}\right)=-\nabla R_{\mu} \cdot \mathbf{u}, \quad x \in \omega_{j} \\
\mathbf{W}_{j}=0, \quad x \in \partial \omega_{j} .
\end{gathered}
$$

Так как

$$
\int_{\omega_{j}} \operatorname{div}\left(R_{\mu} \mathbf{u}\right) d x=\left(1+2^{2 j+2}\right)^{\mu} \int_{S_{2 j+1}} \mathbf{u} \cdot \mathbf{n}^{\prime} d s-\left(1+2^{2 j}\right)^{\mu} \int_{S_{2 j}} \mathbf{u} \cdot \mathbf{n}^{\prime} d s=0
$$

и $\left(1+r^{2}\right) \sim 2^{2 j}$ при $x \in \omega_{j}$, то сушествуют решения $\mathbf{W}_{j}$ задач $(4.38)$, удовлетворяющие неравенствам (см. лемму 2.7)

$$
\begin{aligned}
& \left\|\left(1+r^{2}\right)^{-\mu / 2} \nabla \mathbf{W}_{j} ; L^{2}\left(\omega_{j}\right)\right\| \leqslant c 2^{-j \mu}\left\|\nabla \mathbf{W}_{j} ; L^{2}\left(\omega_{j}\right)\right\| \\
& \quad \leqslant c 2^{-j \mu+j}\left\|\nabla R_{\mu} \cdot \mathbf{u} ; L^{2}\left(\omega_{j}\right)\right\| \leqslant c \mu 2^{-j \mu+j}\left\|\left(1+r^{2}\right)^{\mu-1 / 2} \mathbf{u} ; L^{2}\left(\omega_{j}\right)\right\| \\
& \quad \leqslant c \mu\left\|\left(1+r^{2}\right)^{\mu / 2} \mathbf{u} ; L^{2}\left(\omega_{j}\right)\right\| \leqslant c \mu\left\|\left(1+r^{2}\right)^{\mu / 2} \nabla \mathbf{u} ; L^{2}\left(\omega_{j}\right)\right\| .
\end{aligned}
$$

Далее считаем функции $\mathbf{W}_{j}$ продолженными нулем в $\Omega \backslash \omega_{j}, j=1, \ldots, k-1$.

В результате такой подстановки тождество (4.22) принимает вид

$$
\begin{aligned}
\nu \int_{\Omega} R_{\mu}|\nabla \mathbf{u}|^{2} d x= & -\nu \int_{\Omega} \nabla \mathbf{u} \cdot \mathbf{u} \cdot \nabla R_{\mu} d x-\nu \sum_{j=1}^{k-1} \int_{\omega_{j}} \nabla \mathbf{u} \cdot \nabla \mathbf{W}_{j} d x \\
& -\int_{\Omega}((\mathbf{A} \cdot \nabla) \mathbf{u}+(\mathbf{u} \cdot \nabla) \mathbf{A}+(\mathbf{u} \cdot \nabla) \mathbf{u}) \cdot R_{\mu} \mathbf{u} d x \\
& -\sum_{j=1}^{k-1} \int_{\omega_{j}}((\mathbf{A} \cdot \nabla) \mathbf{u}+(\mathbf{u} \cdot \nabla) \mathbf{A}+(\mathbf{u} \cdot \nabla) \mathbf{u}) \cdot \mathbf{W}_{j} d x \\
& +\int_{\Omega} \mathbf{f}_{1} \cdot R_{\mu} \mathbf{u} d x+\sum_{j=1}^{k-1} \int_{\omega_{j}} \mathbf{f}_{1} \cdot \mathbf{W}_{j} d x .
\end{aligned}
$$


Оценим правую часть (4.40). В силу неравенств Гёльдера, Фридрихса и (4.37), (4.39) получим

$$
\begin{aligned}
& \nu\left|\int_{\Omega} \nabla \mathbf{u} \cdot \mathbf{u} \cdot \nabla R_{\mu} d x\right| \\
& \leqslant c \mu\left(\int_{\Omega_{k} \backslash \omega_{0}}\left(1+r^{2}\right)^{\mu}|\nabla \mathbf{u}|^{2} d x\right)^{1 / 2}\left(\int_{\Omega_{k} \backslash \omega_{0}}\left(1+r^{2}\right)^{\mu}|\mathbf{u}|^{2} d x\right)^{1 / 2} \\
& \quad \leqslant c \mu \int_{\Omega_{k} \backslash \omega_{0}}\left(1+r^{2}\right)^{\mu}|\nabla \mathbf{u}|^{2} d x \leqslant c \mu \int_{\Omega} R_{\mu}|\nabla \mathbf{u}|^{2} d x \\
& \nu\left|\sum_{j=1}^{k-1} \int_{\omega_{j}} \nabla \mathbf{u} \cdot \nabla \mathbf{W}_{j} d x\right| \leqslant \nu \sum_{j=1}^{k-1}\left(\int_{\omega_{j}}\left(1+r^{2}\right)^{\mu}|\nabla \mathbf{u}|^{2} d x\right)^{1 / 2} \\
& \quad \times\left(\int_{\omega_{j}}\left(1+r^{2}\right)^{-\mu}\left|\nabla \mathbf{W}_{j}\right|^{2} d x\right)^{1 / 2} \leqslant c \mu \sum_{j=1}^{k-1} \int_{\omega_{j}}\left(1+r^{2}\right)^{\mu}|\nabla \mathbf{u}|^{2} d x \\
& =c \mu \int_{\Omega} R_{\mu}|\nabla \mathbf{u}|^{2} d x .
\end{aligned}
$$

Кроме того, если $\mu \leqslant 1 / 2$, то, рассуждая так же, как при оценке слагаемого $J_{21}$ в доказательстве леммы 4.5, и используя неравенство (4.39), находим, что

$$
\begin{aligned}
& \left|\int_{\Omega}((\mathbf{A} \cdot \nabla) \mathbf{u}+(\mathbf{u} \cdot \nabla) \mathbf{A}) \cdot R_{\mu} \mathbf{u} d x\right|=\left|\int_{\Omega}\left((\mathbf{A} \cdot \nabla) \mathbf{u} \cdot R_{\mu} \mathbf{u}-(\mathbf{u} \cdot \nabla)\left(R_{\mu} \mathbf{u}\right) \cdot \mathbf{A}\right) d x\right| \\
& \leqslant c\left\|\mathbf{h} ; W^{3 / 2,2}(\partial \Omega)\right\| \int_{\Omega}\left(1+r^{2}\right)^{-1 / 2}\left(R_{\mu}|\mathbf{u}|^{2}+R_{\mu}|\nabla \mathbf{u}|^{2}+|\mathbf{u}|\left|\nabla\left(R_{\mu} \mathbf{u}\right)\right|\right) d x \\
& \leqslant c\left\|\mathbf{h} ; W^{3 / 2,2}(\partial \Omega)\right\| \int_{\Omega}\left(1+r^{2}\right)^{\mu-1 / 2}|\nabla \mathbf{u}|^{2} d x \\
& \leqslant c\left\|\mathbf{h} ; W^{3 / 2,2}(\partial \Omega)\right\| \int_{\Omega}|\nabla \mathbf{u}|^{2} d x \leqslant c_{11}(d), \\
& \left|\sum_{j=1}^{k-1} \int_{\omega_{j}}\left((\mathbf{A} \cdot \nabla) \mathbf{u} \cdot \mathbf{W}_{j}-(\mathbf{u} \cdot \nabla) \mathbf{W}_{j} \cdot \mathbf{A}\right) d x\right| \\
& \leqslant c\left\|\mathbf{h} ; W^{3 / 2,2}(\partial \Omega)\right\| \sum_{j=1}^{k-1}\left(\int_{\omega_{j}}\left(1+r^{2}\right)^{\mu-1}\left(|\mathbf{u}|^{2}+|\nabla \mathbf{u}|^{2}\right) d x\right)^{1 / 2} \\
& \times\left(\int_{\omega_{j}}\left(1+r^{2}\right)^{-\mu}\left(\left|\nabla \mathbf{W}_{j}\right|^{2}+\left|\mathbf{W}_{j}\right|^{2}\right) d x\right)^{1 / 2} \\
& \leqslant c \mu\left\|\mathbf{h} ; W^{3 / 2,2}(\partial \Omega)\right\| \sum_{j=1}^{k-1}\left(\int_{\omega_{j}}\left(1+r^{2}\right)^{\mu-1}|\nabla \mathbf{u}|^{2} d x\right)^{1 / 2} \\
& \times\left(\int_{\omega_{j}}\left(1+r^{2}\right)^{\mu}|\nabla \mathbf{u}|^{2} d x\right)^{1 / 2}
\end{aligned}
$$




$$
\begin{aligned}
& \leqslant c \mu\left\|\mathbf{h} ; W^{3 / 2,2}(\partial \Omega)\right\| \sum_{j=1}^{k-1} 2^{-j}\left(\int_{\omega_{j}}\left(1+r^{2}\right)^{\mu-1 / 2}|\nabla \mathbf{u}|^{2} d x\right)^{1 / 2} \\
& \quad \times 2^{j}\left(\int_{\omega_{j}}\left(1+r^{2}\right)^{\mu-1 / 2}|\nabla \mathbf{u}|^{2} d x\right)^{1 / 2} \\
& \leqslant c\left\|\mathbf{h} ; W^{3 / 2,2}(\partial \Omega)\right\| \int_{\Omega}\left(1+r^{2}\right)^{\mu-1 / 2}|\nabla \mathbf{u}|^{2} d x \leqslant c_{12}(d) .
\end{aligned}
$$

Наконец, легко видеть, что

$$
\begin{gathered}
\int_{\Omega} \mathbf{f}_{1} \cdot R_{\mu} \mathbf{u} d x \leqslant c \delta \int_{\Omega} R_{\mu}|\nabla \mathbf{u}|^{2} d x+c_{\delta} \int_{\Omega} R_{\mu}\left|\mathbf{f}_{1}\right|^{2} d x \\
\leqslant c \delta \int_{\Omega} R_{\mu}|\nabla \mathbf{u}|^{2} d x+c_{\delta} \int_{\Omega}\left(1+r^{2}\right)^{\mu}\left|\mathbf{f}_{1}\right|^{2} d x \\
\leqslant c \delta \int_{\Omega} R_{\mu}|\nabla \mathbf{u}|^{2} d x+c_{\delta} \int_{\Omega}\left(1+r^{2}\right)^{\beta_{1}+2}\left|\mathbf{f}_{1}\right|^{2} d x \\
\sum_{j=1}^{k-1} \int_{\omega_{j}} \mathbf{f}_{1} \cdot \mathbf{W}_{j} d x \leqslant c \sum_{j=1}^{k-1}\left(\int_{\omega_{j}}\left(1+r^{2}\right)^{\mu}\left|\mathbf{f}_{1}\right|^{2} d x+\int_{\omega_{j}}\left(1+r^{2}\right)^{-\mu}\left|\nabla \mathbf{W}_{j}\right|^{2} d x\right) \\
\leqslant c \int_{\Omega}\left(1+r^{2}\right)^{\beta_{1}+2}\left|\mathbf{f}_{1}\right|^{2} d x+c \mu^{2} \sum_{j=1}^{k-1} \int_{\omega_{j}}\left(1+r^{2}\right)^{\mu}|\nabla \mathbf{u}|^{2} d x \\
\leqslant c \int_{\Omega}\left(1+r^{2}\right)^{\beta_{1}+2}\left|\mathbf{f}_{1}\right|^{2} d x+c \mu^{2} \int_{\Omega} R_{\mu}|\nabla \mathbf{u}|^{2} d x .
\end{gathered}
$$

Подставим полученные неравенства в (4.40). Это дает

$$
\begin{aligned}
& \nu \int_{\Omega} R_{\mu}|\nabla \mathbf{u}|^{2} d x \leqslant c(\delta+\mu) \int_{\Omega} R_{\mu}|\nabla \mathbf{u}|^{2} d x+c_{13}(d) \\
& \quad+\left|\int_{\Omega}(\mathbf{u} \cdot \nabla) \mathbf{u} \cdot R_{\mu} \mathbf{u} d x\right|+\left|\sum_{j=1}^{k-1} \int_{\omega_{j}}(\mathbf{u} \cdot \nabla) \mathbf{u} \cdot \mathbf{W}_{j} d x\right| .
\end{aligned}
$$

Оценим два последних слагаемых в правой части (4.41). Поскольку

$$
\int_{\Omega}(\mathbf{u} \cdot \nabla) \mathbf{u} \cdot R_{\mu} \mathbf{u} d x=-\int_{\Omega}|\mathbf{u}|^{2} \mathbf{u} \cdot \nabla R_{\mu} d x
$$

TO

$$
\begin{aligned}
\left|\int_{\Omega}(\mathbf{u} \cdot \nabla) \mathbf{u} \cdot R_{\mu} \mathbf{u} d x\right| & \leqslant \int_{\Omega}|\mathbf{u}|^{3}\left|\nabla R_{\mu}\right| d x \leqslant c \int_{\Omega}\left(1+r^{2}\right)^{\mu-1 / 2}|\mathbf{u}|^{3} d x \\
& \leqslant c\left(\int_{\Omega}|\nabla \mathbf{u}|^{2} d x\right)^{3 / 2} \leqslant c_{14}(d) .
\end{aligned}
$$


Далее, в силу (4.39) и (4.40) имеет место оценка

$$
\begin{aligned}
& \left|\sum_{j=1}^{k-1} \int_{\omega_{j}}(\mathbf{u} \cdot \nabla) \mathbf{u} \cdot \mathbf{W}_{j} d x\right| \\
& \quad \leqslant \sum_{j=1}^{k-1}\left(\int_{\omega_{j}}\left(1+r^{2}\right)^{\mu}|\mathbf{u}|^{2}|\nabla \mathbf{u}|^{2} d x\right)^{1 / 2}\left(\int_{\omega_{j}}\left(1+r^{2}\right)^{-\mu}\left|\mathbf{W}_{j}\right|^{2} d x\right)^{1 / 2} \\
& \quad \leqslant c \mu \sup _{x \in \bar{\Omega}}\left(|\mathbf{u}(x)|^{2}\right) \sum_{j=1}^{k-1} \int_{\omega_{j}}\left(1+r^{2}\right)^{\mu}|\nabla \mathbf{u}|^{2} d x \\
& \quad \leqslant c c_{4}(d) \mu \int_{\Omega_{k} \backslash \omega_{0}}\left(1+r^{2}\right)^{\mu}|\nabla \mathbf{u}|^{2} d x \leqslant c_{15}(d) \mu \int_{\Omega} R_{\mu}|\nabla \mathbf{u}|^{2} d x
\end{aligned}
$$

Таким образом, из (4.41) следует, что

$$
\nu \int_{\Omega} R_{\mu}(x, k)|\nabla \mathbf{u}|^{2} d x \leqslant\left(c_{*}(\delta+\mu)+c_{15}(d) \mu\right) \int_{\Omega} R_{\mu}(x, k)|\nabla \mathbf{u}|^{2} d x+c_{16}(d)
$$

причем постоянные не зависят от $k$. Выберем $\delta$ и $\widehat{\beta}$ так, чтобы выполнялись соотношения

$$
c_{*} \widehat{\beta}+c_{15}(d) \widehat{\beta}=\nu / 4, \quad c_{*} \delta=\nu / 4
$$

Тогда для любого $\mu \in\left(0, \beta_{*}\right), \beta_{*}=\min (1 / 2, \widehat{\beta})$, из $(4.42)$ следует оценка

$$
\int_{\Omega_{k}}\left(1+r^{2}\right)^{\mu}|\nabla \mathbf{u}|^{2} d x \leqslant c \int_{\Omega} R_{\mu}|\nabla \mathbf{u}|^{2} d x \leqslant c_{17}(d)
$$

Переходя в (4.43) к пределу при $k \rightarrow \infty$, находим, что

$$
\int_{\Omega}\left(1+r^{2}\right)^{\mu}|\nabla \mathbf{u}|^{2} d x \leqslant c_{17}(d) \quad \forall \mu \in\left(0, \beta_{*}\right)
$$

Лемма доказана.

ЗАмЕчАниЕ 4.1. Из доказательства леммы 4.6 следует, что величина $\beta_{*}$ зависит от норм правых частей $\mathbf{f}$ и $\mathbf{h}$, причем

$$
\beta_{*} \rightarrow 0 \text { при }\left\|\mathbf{h} ; W^{3 / 2,2}(\partial \Omega)\right\|+\left\|\mathbf{f} ; L_{\beta_{1}+2}^{2}(\Omega)\right\| \rightarrow \infty .
$$

Лемма 4.7. Пусть выполнены условия леммы 4.5. Тогда для обобщенного решения задачи (4.20) имеет место оценка

$$
\left\|\mathbf{u} ; C_{\mu}^{0}(\bar{\Omega})\right\| \leqslant c_{18}(d)
$$


ДоказАтельство. Рассмотрим и как обобщенное решение системы НавьеСтокса (3.1) с правой частью $\left(\mathbf{f}_{1}-(\mathbf{A} \cdot \nabla) \mathbf{u}-(\mathbf{u} \cdot \nabla) \mathbf{A}, 0,0\right)$. Из (4.11) следует, что

$$
\begin{aligned}
\left\|\mathbf{u} ; C^{0}\left(\bar{Q}_{k, s}\right)\right\| & \leqslant c\left(\left\|\mathbf{u} ; W^{1,2}\left(Q_{k, s}^{*}\right)\right\|^{4}+\left\|\mathbf{f}_{1}-(\mathbf{A} \cdot \nabla) \mathbf{u}-(\mathbf{u} \cdot \nabla) \mathbf{A} ; L^{2}\left(Q_{k, s}^{*}\right)\right\|\right) \\
& \leqslant c\left(\left\|\nabla \mathbf{u} ; L^{2}\left(Q_{k, s}^{*}\right)\right\|^{4}+\left\|\mathbf{f}_{1} ; L^{2}\left(Q_{k, s}^{*}\right)\right\|+c_{19}(d)\left\|\nabla \mathbf{u} ; L^{2}\left(Q_{k, s}^{*}\right)\right\|\right) .
\end{aligned}
$$

Умножим $(4.45)$ на $\left(1+\left(k^{2}+s^{2}\right)\right)^{\mu / 2}$ ивоспользуемся тем, что $\left(1+\left(k^{2}+s^{2}\right)\right) \sim\left(1+r^{2}\right)$ при $x \in Q_{k, s}^{*}$. В силу (4.36) это дает

$$
\begin{aligned}
\|(1+ & \left.r^{2}\right)^{\mu / 2} \mathbf{u} ; C^{0}\left(\bar{Q}_{k, s}\right) \| \leqslant c\left(\left\|\left(1+r^{2}\right)^{\mu / 8} \nabla \mathbf{u} ; L^{2}\left(Q_{k, s}^{*}\right)\right\|^{4}\right. \\
& \left.+\left\|\left(1+r^{2}\right)^{\mu / 2} \mathbf{f}_{1} ; L^{2}\left(Q_{k, s}^{*}\right)\right\|+c_{19}(d)\left\|\left(1+r^{2}\right)^{\mu / 2} \nabla \mathbf{u} ; L^{2}\left(Q_{k, s}^{*}\right)\right\|\right) \\
& \leqslant c\left(\left\|\nabla \mathbf{u} ; L_{\mu}^{2}(\Omega)\right\|^{4}+c_{19}(d)\left\|\nabla \mathbf{u} ; L_{\mu}^{2}(\Omega)\right\|+\left\|\mathbf{f}_{1} ; L_{\mu}^{2}(\Omega)\right\|\right) \leqslant c_{20}(d) .
\end{aligned}
$$

Взяв в последнем неравенстве супремум по всем $k, s \in \mathbb{Z}$, выводим (4.44). Лемма доказана.

Докажем теперь основной результат работы.

Tеорема 4.2. Пyсm $\mathbf{f} \mathbf{f} \in \mathcal{V}_{\beta_{1}+2, l-1}^{l-1}(\Omega), l \geqslant 1, \beta_{1} \in(-1,0), \mathbf{h} \in W^{l+3 / 2,2}(\partial \Omega)$ $u$

$$
\operatorname{supp} \mathbf{h} \subset \partial \Omega \cap B_{R_{0}}, \quad \int_{\partial \Omega} \mathbf{h} \cdot \mathbf{n} d s=F \neq 0 .
$$

Тогда существует решение $(\mathbf{v}, p)$ задачи (3.1), для которого справедливо асимптотическое представление

$$
\left(\begin{array}{c}
\mathbf{v} \\
p
\end{array}\right)=\left(\begin{array}{c}
\chi(r) 3 F \pi^{-1} z(z-1)\left(y / r^{2}, 0\right) \\
\text { const }+\chi(r) 6 \nu F \pi^{-1} \ln r
\end{array}\right)+\left(\begin{array}{c}
\mathbf{u} \\
q
\end{array}\right)
$$

əde $(\mathbf{u}, q) \in \mathscr{D}_{\beta_{1}}^{l}(\Omega) u$

$$
\left\|(\mathbf{u}, q) ; \mathscr{D}_{\beta_{1}}^{l}(\Omega)\right\| \leqslant c_{21}(d)
$$

ДокАЗАТЕЛЬСтво. Пусть $\mathbf{v}$ - обобшенное решение задачи (3.1) (см. теорему 4.1). Представим v в виде

$$
\mathbf{v}=\mathbf{A}+\mathbf{u}
$$

где $\mathbf{u}$ - решение задачи (4.20). В силу лемм 4.6, 4.7

$$
\begin{gathered}
\int_{\Omega}\left(1+r^{2}\right)^{\mu}|\nabla \mathbf{u}|^{2} d x<\infty, \\
\sup _{x \in \bar{\Omega}}\left(\left(1+r^{2}\right)^{\mu / 2}|\mathbf{u}(x)|\right)<\infty \quad \forall \mu \in\left(0, \beta_{*}\right), \quad \beta_{*} \in(0,1 / 2) .
\end{gathered}
$$

Рассмотрим нелинейное слагаемое

$$
(\mathbf{v} \cdot \nabla) \mathbf{v}=(\mathbf{A} \cdot \nabla) \mathbf{A}+(\mathbf{u} \cdot \nabla) \mathbf{A}+(\mathbf{A} \cdot \nabla) \mathbf{u}+(\mathbf{u} \cdot \nabla) \mathbf{u}
$$


Используя соотношения (см. лемму 4.4)

$$
\left|\partial_{y}^{\gamma} \partial_{z}^{\alpha} \mathbf{A}(x)\right| \leqslant c|F|\left(1+r^{2}\right)^{-(1+|\gamma|) / 2}, \quad A_{3}(x)=0,
$$

справедливые при $r \geqslant 3 R_{0}$, находим, что $(\mathbf{A} \cdot \nabla) \mathbf{A} \in \mathscr{V}_{\beta_{1}+2, l-1}^{l-1}(\Omega)$. Далее, при $r \geqslant 3 R_{0}$

$$
\begin{aligned}
|(\mathbf{A} \cdot \nabla) \mathbf{u}+(\mathbf{u} \cdot \nabla) \mathbf{A}| & \leqslant(|\mathbf{A}|+|\nabla \mathbf{A}|)(|\mathbf{u}|+|\nabla \mathbf{u}|) \\
& \leqslant c|F|\left(1+r^{2}\right)^{-1 / 2}(|\mathbf{u}|+|\nabla \mathbf{u}|)
\end{aligned}
$$

и, следовательно, $(\mathbf{A} \cdot \nabla) \mathbf{u}+(\mathbf{u} \cdot \nabla) \mathbf{A} \in L_{\mu+1}^{2}(\Omega)$.

Кроме того, в силу (4.44)

$$
\begin{aligned}
|(\mathbf{u} \cdot \nabla) \mathbf{u}| & \leqslant c \sup _{x \in \bar{\Omega}}\left(\left(1+r^{2}\right)^{\mu / 2}|\mathbf{u}(x)|\right)\left(1+r^{2}\right)^{-\mu / 2}|\nabla \mathbf{u}(x)| \\
& \leqslant c_{22}(d)\left(1+r^{2}\right)^{-\mu / 2}|\nabla \mathbf{u}(x)| \in L_{2 \mu}^{2}(\Omega) .
\end{aligned}
$$

Таким образом, $(\mathbf{v} \cdot \nabla) \mathbf{v} \in L_{2 \mu}^{2}(\Omega)$. Из (4.48) следует, что

$$
\mathbf{u}^{\prime} \in \mathscr{V}_{\beta+1,1}^{1}(\Omega), \quad u_{3} \in \mathscr{V}_{\beta+2,0}^{1}(\Omega), \quad \beta=\mu-2 \in(-2,-3 / 2) .
$$

Отметим, что здесь мы загрубили показатель весового множителя в слагаемом $\int_{\Omega}\left(1+r^{2}\right)^{\beta+1}\left(\left|\mathbf{u}^{\prime}\right|^{2}+\left|\partial_{z} \mathbf{u}^{\prime}\right|^{2}\right) d x$ для того, чтобы погрузить $\mathbf{u}^{\prime}$ в пространство $\mathscr{V}_{\beta+1,1}^{1}(\Omega)$ с анизотропным распределением веса (на самом деле, $\mathbf{u}^{\prime}, \partial_{z} \mathbf{u}^{\prime} \in$ $\left.L_{\beta+2}^{2}(\Omega)\right)$.

В лемме 4.4 доказано включение $\mathbf{A}^{\prime} \in \mathscr{V}_{-\varepsilon, l+1}^{l+1}(\Omega) \forall \varepsilon>0$. Взяв $\varepsilon=1-\mu>0$, получим $\mathbf{A}^{\prime} \in \mathscr{V}_{\beta+1, l+1}^{l+1}(\Omega)$. Так как $A_{3}(x)=0$ при $r \geqslant 3 R_{0}$, то отсюда выводим, что

$$
\mathbf{v}^{\prime} \in \mathscr{V}_{\beta+1,1}^{1}(\Omega), \quad v_{3} \in \mathscr{V}_{\beta+2,0}^{1}(\Omega) .
$$

По теореме 3.1 существует функция $p \in L_{\beta}^{2}(\Omega)$ такая, что пара $(\mathbf{v}, p)$ удовлетворяет интегральному тождеству (3.5). Представим $p$ в виде

$$
p(x)=q(x)+\chi(r) \frac{6 \nu F}{\pi} \ln r .
$$

Легко видеть, что $q(x)=p(x)-\chi(r) 6 \nu F \pi^{-1} \ln r \in L_{\beta}^{2}(\Omega)$.

Возможны два случая:
a) $\beta=\mu-2 \in\left(-\frac{5}{3},-\frac{3}{2}\right)$,
b) $\beta \in\left(-2,-\frac{5}{3}\right)$.

В случае а) рассмотрим $(\mathbf{v}, p) \in L_{\beta+1}^{2}(\Omega) \times L_{\beta}^{2}(\Omega)$ как решение линейной задачи Стокса $(2.23)$ с правой частью $(\mathbf{f}-(\mathbf{v} \cdot \nabla) \mathbf{v}, 0, \mathbf{h}) \in \mathscr{R}_{\beta}^{1}(\Omega ; \partial \Omega)$. По теореме 2.1 (i) $(\mathbf{v}, p) \in \mathscr{D}_{\beta}^{1}(\Omega)$, т.е.

$$
\mathbf{v}^{\prime} \in \mathscr{V}_{\beta+1,1}^{2}(\Omega), \quad v_{3} \in \mathscr{V}_{\beta+2,0}^{2}(\Omega)
$$


Поскольку $\beta>-5 / 3$, то в силу замечания 3.2 этих включений достаточно для справедливости теоремы 3.2 и, следовательно, $(\mathbf{v}, p)$ допускает асимптотическое представление $(4.46)$ с $(\mathbf{u}, q) \in \mathscr{D}_{\beta_{1}}^{l}(\Omega)$.

Рассмотрим случай b) и сведем его к случаю а). Функции и и $q$, удовлетворяющие системе (4.20), будем интерпретировать как решение задачи Стокса (2.23) с правой частью $\left(\mathbf{f}_{1}-(\mathbf{A} \cdot \nabla) \mathbf{u}-(\mathbf{u} \cdot \nabla) \mathbf{A}-(\mathbf{u} \cdot \nabla) \mathbf{u}, 0, \mathbf{h}\right)$. По доказанному $(\mathbf{v} \cdot \nabla) \mathbf{v} \in L_{2 \mu}^{2}(\Omega)$. Следовательно,

$$
\left(\mathbf{f}_{1}-(\mathbf{A} \cdot \nabla) \mathbf{u}-(\mathbf{u} \cdot \nabla) \mathbf{A}-(\mathbf{u} \cdot \nabla) \mathbf{u}, 0, \mathbf{h}\right) \in \mathscr{R}_{\beta+\mu}^{1}(\Omega ; \partial \Omega)
$$

и согласно теореме 2.1 (i) $(\mathbf{u}, q) \in \mathscr{D}_{\beta+\mu}^{1}(\Omega)$. Это дает

$$
\mathbf{u}^{\prime} \in \mathscr{V}_{\beta+1+\mu, 1}^{2}(\Omega), \quad u_{3} \in \mathcal{V}_{\beta+2+\mu, 0}^{2}(\Omega)
$$

Если $2 \mu-2>-5 / 3$, то выполнены условия случая а) и теорема доказана. Если же $2 \mu-2<-5 / 3$, то упомянутые включения влекут конечность интеграла $\int_{\Omega}\left(1+r^{2}\right)^{2 \mu}|\nabla \mathbf{u}|^{2} d x$ и из леммы 4.7 находим, что $\mathbf{u} \in C_{2 \mu}^{0}(\bar{\Omega})$. Повторяя вышеизложенные рассуждения, заключаем, что $\mathbf{f}_{1}-(\mathbf{A} \cdot \nabla) \mathbf{u}-(\mathbf{u} \cdot \nabla) \mathbf{A}-(\mathbf{u} \cdot \nabla) \mathbf{u} \in L_{\beta+2 \mu}^{2}(\Omega)$. То есть

$$
\left(\mathbf{f}_{1}-(\mathbf{A} \cdot \nabla) \mathbf{u}-(\mathbf{u} \cdot \nabla) \mathbf{A}-(\mathbf{u} \cdot \nabla) \mathbf{u}, 0, \mathbf{h}\right) \in \mathscr{R}_{\beta+2 \mu}^{1}(\Omega ; \partial \Omega)
$$

и теорема 2.1 (i) дает включение $(\mathbf{u}, q) \in \mathscr{D}_{\beta+2 \mu}^{1}(\Omega)$. Таким образом, за конечное число шагов случай b) сводится к случаю а). Теорема доказана.

\section{Список литературы}

1. Heywood J. G. On uniqueness question in the theory of viscous flow // Acta Math. 1976. V. 136. P. $61-102$.

2. Капитанский Л. В., Пилецкас К.И. О пространствах соленоидальных векторных полей и краевых задачах для уравнений Навье-Стокса в областях с некомпактньми границами // Труды МИАН. 1983. Т. 159. С. 5-36.

3. Ладыженская О.А., Солонников В. А. О некоторых задачах векторного анализа и об обобшенных постановках краевых задач для уравнений Навье-Стокса // Записки научн. сем. ЛОМИ. 1976. Т. 59. С. 81-116.

4. Ладьженская $O . A$., Солонников B. A. О разрешимости краевых и начально-краевых задач для уравнений Навье-Стокса в областях с некомпактньми границами // Вестн. ЛГУ. 1977. № 13. С. 39-47.

5. Ладыженская О.А., Солонников B.A. О нахождении решений краевых задач для стационарных уравнений Стокса и Навье-Стокса, имеющих неограниченный интеграл Дирихле // Записки научн. сем. ЛОМИ. 1980. Т. 96. С. 117-160.

6. Nazarov S. A., Pileckas K. Asymptotics of solutions to Stokes and Navier-Stokes equations in domains with paraboloidal outlets to infinity // Rend. Sem. Mat. Univ. Padova. 1998. V. 99. P. 1-43.

7. Пилецкас К.И. О существовании решений уравнений Навье-Стокса, имеющих бесконечную диссипацию энергии, в одном классе областей с некомпактной границей // Записки научн. сем. ЛОМИ. 1981. Т. 110. С. 180-202.

8. Солонников B. А., Пилецкас K.И. О некоторых пространствах соленоидальных векторов и о разрешимости краевой задачи для системы уравнений Навье-Стокса в областях с некомпактньми границами // Записки научн. сем. ЛОМИ. 1977. Т. 73. С. 136-151.

9. Solonnikov V.A. On the solvability of boundary and initial-boundary value problems for the Navier-Stokes system in domains with noncompact boundaries // Pacific J. Math. 1981. V. 93. № 2. P. 443-458. 
10. Солонников В. А. О решениях стационарных уравнений Навье-Стокса с бесконечньм интегралом Дирихле // Записки научн. сем. ЛОМИ. 1982. Т. 115. С. 257-263.

11. Solonnikov V. A. Stokes and Navier-Stokes equations in domains with noncompact boundaries // Nonlinear partial differential equations and their applications. Coll. France Semin. 1983. V. 4. Res. Notes Math. V. 84. P. 240-349.

12. Solonnikov V.A. Boundary and initial-boundary value problems for the Navier-Stokes equations in domains with noncompact boundaries // Mathematical topics in fluid mechanics. Proc. of the summer course. Lisbon, Portugal, September 9-13, 1991 / ed. J. F. Rodrigues et al. Harlow, Essex: Longman, 1993. P. 117-162.

13. Pileckas $K$. Weighted $L^{q}$-solvability for the steady Stokes system in domains with noncompact boundaries // Math. Models Methods Appl. Sci. 1996. V. 6. № 1. P. 97-136.

14. Pileckas $K$. Classical solvability and uniform estimates for the steady Stokes system in domains with noncompact boundaries // Math. Models Methods Appl. Sci. 1996. V. 6. № 2. P. 149-167.

15. Pileckas $K$. Strong solutions of the steady nonlinear Navier-Stokes system in domains with exits to infinity // Rend. Sem. Mat. Univ. Padova. 1997. V. 97. P. 236-267.

16. Pileckas $K$. Recent advances in the theory of Stokes and Navier-Stokes equations in domains with noncompact boundaries // Mathematical theory in fluid mechanics. Lectures of the 4th winter school. Paseky, Czech Republic, December 3-9, 1995 / ed. G.P. Galdi et al. Harlow, Essex: Longman, 1996. P. 30-85.

17. Nazarov S. A., Pileckas K. On the solvability of the Stokes and Navier-Stokes problems in the domains that are layer-like at infinity // J. Math. Fluid Mech. 1999. V. 1. №1. P. 78-116.

18. Nazarov S. A., Pileckas K. The asymptotic properties of the solutions to the Stokes problem in domains that are layer-like at infinity // J. Math. Fluid Mech. 1999. V. 1. № 2 . P. 131-167.

19. Nazarov S. A., Pileckas K. On the Fredholm property of the Stokes operator in a layer-like domain // Z. Anal. Anwendungen. 2001. V. 20. № 1. P. 155-182.

20. Назаров C. А., Пилецкас К. И. Фредгольмовость оператора задачи Неймана в областях с выходом на бесконечность в виде слоя // Алгебра и анализ. 1996. Т. 8. №6. С. 57-104.

21. Капитанский Л.В., Пилецкас К.И. О некоторых задачах векторного анализа // Записки научн. сем. ЛОМИ. 1984. Т. 138. С. 65-85.

22. Лады женская $О$. А. Математические вопросы динамики вязкой несжимаемой жидкости. М.: Наука, 1970.

Институт математики и информатики,

Поступила в редакцию

г. Вильнюс, Литва

E-mail: pileckas@ktl.mii.lt

10.08.2000 и 11.03 .2002 